\title{
Original Paper Synopsis of Capparaceae to the flora of Colombia
}

\author{
Jorge David Mercado-Gómez ${ }^{1,2,5,6}$, Mailyn Adriana González ${ }^{3}$ \& María Eugenia Morales-Puentes ${ }^{4}$
}

\begin{abstract}
A taxonomic synopsis of Capparaceae distributed in Colombia was carried out reviewing 1,800 botanical specimens from 13 herbaria in Colombia, five international institutions and close to 100 field collected specimens. We found 32 species and ten genera according to the last taxonomic modification proposed for this family. From these species, we reevaluated the conventional features used on the species identification, from which detailed description for each species and taxonomic keys were developed. In addition, geographic distribution maps in Colombia, and information about vernacular names and Neotropical distribution was added. Key words: Capparaceae, Neotropic, species, taxa, taxonomy.

Resumo

Foi realizada uma sinopse taxonômica de Capparaceae da Colômbia e sua distribuição com a revisão de 1.800 amostras botânicas de 13 herbários da Colômbia, cinco instituições internacionais e cerca de 100 amostras coletadas em campo. Foram encontradas 32 espécies e oito gêneros de acordo com a última modificação taxonômica proposta para esta família. A partir dessas espécies, reavaliamos as características convencionais usadas na identificação de espécies, a partir das quais se realizou uma ampliação de descrições detalhadas de cada espécie. Além disso, foram adicionados mapas de distribuição geográfica na Colômbia e informações sobre nomes vernáculos e distribuição neotropical.
\end{abstract}

Palavras-chave: Capparaceae, Neotrópico, espécie, taxa, taxonomia.

\section{Introduction}

Capparaceae is a family of approximately 40-45 genera and 700-800 species distributed in tropical and subtropical regions (Short 2011). In the Neotropics 19 genera and 104 species are currently recognized (Cornejo \& Iltis 2008a,b,c,d,e, 2009, 2010c, 2013; Cornejo et al. 2008).

Antoine Laurent de Jussieu described Capparaceae in 1789 based on Capparis, from the Greek Kapparis or Kappari and Latin Capparis, which refers to shrubs or fruits of caper, Capparis spinosa L., (Quattrocchi 2000). After describing Capparis, Jussieu referred to the species of this group using the unofficial name Capparides. In this sense, the family name Capparidae was subsequently adopted in other taxonomic analyses (Bentham \& Hooker 1862; De Candolle 1824) and, finally years later was named Capparidaceae (Bullock 1958, 1959) and accepted by the International Rules of Botanical Nomenclature (Lanjow \& Sprague 1947). However, in 1961 the Montreal code established that the correct name is Capparaceae based on current nomenclatural

\footnotetext{
${ }^{1}$ Universidad de Sucre, Depto. Biología y Química, Grupo Evolución y Sistemática Tropical, Cra. 28 \#5-267, Puerta Roja, Sincelejo, Sucre, Colombia.

${ }^{2}$ Universidad Nacional de Colombia, Depto. Ciencias Forestales, Carrera 65 Nro. 59A-110, Medellín, Colombia.

${ }^{3}$ Instituto de Investigación de Recursos Biológicos Alexander von Humboldt, Lab. Genética de la Conservación, Calle 28a \# 15-09, Bogota D.C, Colombia.

${ }^{4}$ Universidad Pedagógica y Tecnológica de Colombia, Herbario UPTC, Grupo Sistemática Biológica, Av. Central del Norte 39-115, Tunja, Boyacá, Colombia.

${ }^{5}$ ORCID: <https://orcid.org/0000-0002-4619-0028>

${ }^{6}$ Author for correspondence: jorge.mercado@unisucre.edu.co
} 
rules, e.g., dropping -is from Capparis and adding -aceae (International Association for Plant Taxonomy CIdlNB 1961). This modification was rejected by several authors who proposed keeping Capparidaceae because it came from the historical usage of Capparides, but the official name published by Jussieu was Capparis (Crosswhite \& Iltis 1966; Dugand 1968b).

Capparaceae, as Capparidaceae, was divided by De Candolle (1824) into two subtribes Cappareae and Cleomeae, including 231 species and 17 genera, these subtribes were accepted by Bentham \& Hooker (1862) and by Eichler (1865). Subsequently, Pax (1891) changed the rank to subfamily Capparidoideae and Cleomoideae, as well as incorporating other genera as additional subfamilies Dipterygioideae, Emblingioideae, and Roydsioideae. Years later, Pax \& Hoffmann (1936) increased the number of subfamilies to eight (Buhsioideae, Calyptrothecoideae, Capparidoideae, Cleomoideae, Dipterygioideae, Emblingioideae, Pentadiplandroideae, and Podandrogynoideae) and increased the number of genera to 45 , twenty of which were monotypic. In addition, due to the fact that the subfamily Capparidoideae contained the greatest number of species, these authors recognized four subtribes, Capparideae, Koeberlinieae, Maerueae, and Stixeae.

Nevertheless, Hutchinson (1967) in disagreement with the classification of Pax \& Hoffmann (1936) proposed Capparidaceae with 32 genera and 440 species distributed in three subtribes (Apophylleae, Cadabeae, and Capparideae), without recognizing subfamilies. Hutchinson (1967) established this family in the order Capparales. Cronquist (1981) maintained Capparales but changed the name of the family to Capparaceae, divided it into the subfamilies Capparoideae and Cleomoidae, and recognized 45 genera and 800 species. Likewise, Takhtajan (1997) supported the classification of Cronquist (1981) but reduced the number of genera to 37 and increased the species to 900 ; he also insisted on adopting the name Capparaceae. Morton et al. (1997) removed the genus Physena to its own family, Physenaceae, and transferred it to Caryophyllales.

Subsequently, with molecular phylogenetic analyses, the classification system of Capparaceae has changed. A few analyses have found a close relationship between the subtribe Thelypodieae of Brassicaceae and Cleome of Cleomoideae
(Judd et al. 1994). On the basis of these results Capparaceae was reduced to the level of subfamily and was included along with subfamily Cleomoideae in Brassicaceae s.l. Furthermore, other authors also suggested that Cleomoideae were more closely related with Brassicaceae than with Capparaceae (Rodman et al. 1993, 1996). These results led Hall et al. (2002) to analyze the phylogeny of Capparaceae and Brassicaceae using plastid genes, founding that Capparaceae s.l. was paraphyletic with respect to Brassicaceae.

However, evolutionary relationships within Capparaceae s.str. remained unclear, because the study by Hall et al. (2002) had limited taxon sampling. Hall (2008) performed another phylogenetic analysis of Capparaceae using two plastid genes and including 15 more species. The analysis clearly showed that Cleomoideae is more closely related to Brassicaceae with respect to Capparoideae, which led Hall (2008) to suggest a taxonomic revision separating the clades into three families, which were subsequently formally recognized by Iltis et al. (2011) as Capparaceae, Cleomaceae, and Brassicaceae. Another important outcome was that Capparis is paraphyletic, so it was proposed to segregate the genus into smaller clades for nomenclatural stability and monophyly. In this way species of Capparis were segregated into two large lineages, e.g. Capparis s.str. clade, which is predominantly Old World, and a clade of entirely New World Capparis, the latter more closely related to genera Atamisquea, Belencita, and Morisonia. Nonetheless, there are no useful morphological features for recognizing the New World taxa as a single genus. On the basis of these results, Iltis and Cornejo, in several publications, formalized the species of Neotropical capparoids as the following genera: Anisocapparis (1 species), Beautempsia (1) Calanthea (2), Caphexandrea (1), Capparicordis (3), Colicodendron (6), Capparidastrum (19), Cynophalla (16) Hispaniolanthus (1), Mesocapparis (1), Monilicarpa (2), Neocalyptrocalyx (7), Preslianthus (3), Quadrella (25), and Sarcotoxicum (1) (Cornejo et al. 2014; Cornejo 2010; Cornejo \& Iltis 2005b, 2008b, c, e, 2009, 2010a, b, c, d; Galetti et al. 2016; Iltis 1965; Iltis \& Cornejo 2007a, b, 2010a, b). There are no species of Capparis s.str. in the Neotropics.

Over the last few years, several taxonomic monographs of this family have been published, mainly in Central America (Cornejo \& Iltis 2012, 2013). In South America only new species (Cornejo \& Iltis 2005a, 2010d), taxonomic 
combinations (Cornejo \& Iltis 2006, 2008a; Iltis 2005; Rodríguez-Rodríguez et al. 2007) and regionals flora (Soares Neto et al. 2014; Soares Neto \& Jardim 2015) have been published; there has not been modern monograph including South American species. In Colombia, Triana and Planchon (1862) recognized 21 species in Capparideae (Capparaceae), distributed in Cartagena, Santa Marta, and close to the Magdalena River. Subsequently, Dugand (1968a, 1941) published a monograph of the genus Capparis in Colombia, as well as other studies that reported the genus Belencita (Dugand 1944). Cornejo \& Mercado-Gómez (2016) described a new species of Steriphoma. More recently, Aguirre De la Hoz \& Zapata (2017) analyzed the Capparaceae of Atlantico and Iltis \& Cornejo (2016) reported 29 species of Capparaceae in the catalogue of lichens and plants of Colombia (Bernal et al. 2016). However, the latter mainly included species deposited at the herbarium COL, and information about species distribution and identification keys is lacking. For this reason, the goal of this research is to carry out an extensive revision of specimens from all the herbaria of Colombia and several other major institutions in other countries, to make a detailed description, and an identification key focusing on vegetative characters; finally, we recorded the species distributions through the herbarium specimens exsiccata and stablished their geographic distribution.

\section{Materials and Methods}

To perform this study, we studied the nomenclatural types of the 29 species reported in Colombia, its taxonomic history, synonyms and valid name. With this information, we revised the protologues of each species and taxonomic types photography in JPS-Jstor Plant Science. Once we studied these taxonomic entities we reviewed the specimens distributed in Colombia at the national herbaria CAUP, COL, CUVC, FMB, HUA, HEUS, HECASA, ICESI, JAUM, MEDEL, UIS, UPTC, UTMC; and the foreign herbaria through online versions $\mathrm{MO}, \mathrm{K}, \mathrm{NY}, \mathrm{GH}$, and MEXU (all the herbarium acronym were carried out according to Holmgren et al. (1990) and updated based on Thiers (continuously updated).

After the herbarium specimens were reviewed, we used the geographic information from each specimen and elaborated distributions maps for each species of Capparaceae distributed in Colombia, employing Q-GIS ${ }^{\circledR}$ (3.0). We used these dataset and maps to select locations that were mainly visited during 2015 and 2017 to collected plant specimens of Capparaceae.

\section{Results and Discussion}

We found 32 species and ten genera occurring in Colombia, from 1,800 specimens reviewed from herbarium and close of 100 wild collected specimens (supplementary material Table S1, available at <https://doi.org/10.6084/ m9.figshare.10141595.v1>). These species mainly grow in lowlands over tropical dry forest, however some taxa grow in wet, very wet and Andean forest (see distribution and habit below). From 32 species, Cynophalla linearis (Jacq.) J. Presl, Capparidastrum cuatrecasanum (Dugand) Cornejo \& Iltis, Capparidastrum grandiflorum Cornejo \& Iltis, Monilicarpa tenuisiliqua (Jacq.) Cornejo \& Iltis, Morisonia multiflora Triana \& Planch., Steriphoma colombianum Dugand, Steriphoma menispermifolium Cornejo \& J. D. Mercado Gómez are endemic to Colombia.

\section{Capparaceae Juss.}

Trees or shrubs 1-40 m tall; stems glabrous or recovered by simple, stellate, peltate, peltatestellate trichomes on new branch; tuberculous lenticels (orbicular or elongate) present or absent. Extrafloral supraxillary nectaries present only in Cynophalla. Leaves evergreen, simple or composed (trifoliate in Crateva), alternates and arranged in spiral, distichous in Cynophalla; stipules present or absent; petiole similar or unequal length, sessile and subsessile (Cynophalla), angulate or terete, stem glabrous or with simple, stellate, peltate, peltate-stellate trichomes, ventrally canaliculated or smooth; pulvinus mainly present on unequal petioles. Blades coriaceous, chartaceous, membranaceous and papyraceous; ovate, oblong, spathulate, ellipsoidal, oblanceolate, hastate and linear; base rounded, truncate, attenuate, cordate, semicordate, cuneate, even deltoid, apex acute, obtuse, acuminate and retuse; margin entire, undulate and revolute; mainly glabrous above and glabrous or short pilose, stellate, peltate and peltate stellate beneath; venation camptodromous. Inflorescences or solitary flowers, axillar or terminal, racemes, racemes corymbiform, or panicles with 6-50 flowers; subtended for bracts or not; pedicels glabrous or short pilose, stellate, peltate and peltate-stellate; terete or angulate and in some case 
ventrally canaliculated. Flowers actinomorphic, zygomorphic and hermaphroditic. Aestivation of calyx valvate, imbricate, calix uniseriate or biseriate, free or fused, mainly glabrous inside and glabrous or short pilose, stellate, peltate, and peltate-stellate. Petals imbricate, fused or free, white, black, cream, greenish and purple, mainly glabrous inside and glabrous. Floral nectaries episepals glands, fleshy, bulbous ovoid or scales deltoid, glabrous mainly or stellate and peltate. Stamens $4-\infty$, inserts or exserts, filaments glabrous or short-pilose at the base; anther basifixed or dorsifixed. Gynophore developed or not (Quadrella odoratissima), exsert, rarely insert, glabrous or short-pilose, stellate and peltate; ovary (sub)sessile, glabrous or short-pilose, stellate, stellate-peltate, peltate, truncate or capitate. Fruits capsular, pepo amphisarca, fleshy or dried, torulous or not, exocarp smooth, verrucose and peltate; seeds variable in number, globoids, ovoid, elliptic or reniform; testa smooth, thick and hard, to thin and easily movable.

\section{Identification key to the genera of Colombian Capparaceae}

1. Plants with leaves compound, trifoliate.

1'. Plants with simple leaves

2. Inflorescences conformed by a solitary flowers

$2^{\prime}$. Inflorescences racemes, corymbose racemes, or panicles ....................................................... 3

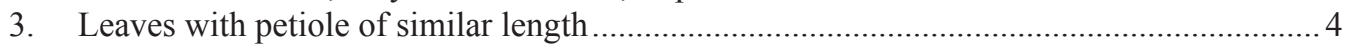

4. Leaves arranged spirally and supraxillary extrafloral nectaries absent........................5

5. Leaves stellate, peltate-stellate beneath. Coriaceous or membranaceous, margin entire......

6. Leaves stellate beneath, and fruits amphisarca, spherical to oblong............. 7

7. Leaves with margin entire, endemic of Colombian Caribbean

7'. Leaves with margin revolute, plants habit in humid and Andean forest.

8. Preslianthus

6'. Leaves peltate-stellate beneath and fruits torulous, capsule linear or cylindric

9. Quadrella

5'. Leaves glabrous in both sides. Chartaceous, margin strongly undulate.

4'. Leave arranged distichous and supraxillary extrafloral nectaries present.

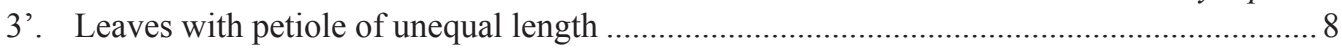

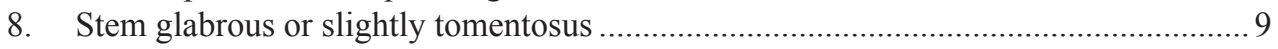

9. Stem without subulate stipules and tuberculous lenticels present.

9'. Stem with subulate stipules and tuberculous lenticels absent

8'. Stem stellate or peltate

10. Stem stellate, flowers zygomorphic. Stamens exsert and ovary smooth.

10'. Stem peltate-stellate, flowers actinomorphic. Stamens insert and ovary ribbed.....

\section{Belencita $\mathrm{H}$. Karst.}

1.1. Belencita nemorosa (Jacq.) Dugand, Caldasia 2: 371. 1944. Capparis nemorosa Jacq., Enum. Syst. Pl. 24. 1760.

Trees or shrubs, $1-5 \mathrm{~m}$ tall; stem usually glabrous, branch with indument stellate-glandular in juvenile and in terminal branch. Leaves simple, alternated and arranged spirally, petiole angular, ventrally canaliculated, of similar length 7-11 mm long, stellate or glandular. Blades coriaceous to chartaceous, ovate to elliptic, $9-12 \times 3.9-4.5 \mathrm{~cm}$, rounded at base, acute, attenuate to semicordate at apex, margin undulate, stellate in both side on new leaves, when mature, glabrous above or sparsely 
stellate in the base of principal nerves and stellate beneath; 7-12 pairs of secondary veins. Solitary flower. Flowers axillar or terminal, actinomorphic and hermaphroditic, subtended by small triangular bracts, densely stellate; pedicel terete, 4-8 $\mathrm{mm}$ long, densely stellate throughout, but glabrous in senescence. Sepals fused, $20 \times 10 \mathrm{~mm}$, lobes deltoid, $15 \times 10 \mathrm{~mm}$, acute to acuminate at apex, stellate in both sides. Floral nectaries scales deltoid and glabrous. Petals imbricate, rhomboid, white, $40 \times 15 \mathrm{~mm}$, acute at apex, inner side stellate and glabrous on the outside. Stamens 8, filaments insert, $20 \mathrm{~mm}$ long, glabrous; anthers basifixed $5.5 \mathrm{~mm}$ long. Gynophore $21.5 \mathrm{~mm}$ long, slightly exserted, glabrous; ovary oblongoid, 5-8 × $2 \mathrm{~mm}$, glabrous; stigma sessile and truncated. Fruits amphisarca, globoid to ovoid 5-6 × $3.54 \mathrm{~cm}$; seeds 6-12, ellipsoidal, globoids to reniform.

Selected specimens examined: COLOMBIA. ATLÁNTICO: Barranquilla, Ciénega de Mallorquin,

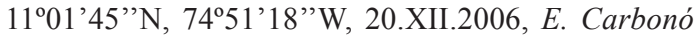
4508 (UTMC). MAGDALENA: San Zenon, Pijiño, 09¹5'00'N, 74²5'00'W, 10.VI.1993, J.G. Ramírez 4642 (JAUM).

Endemic to the Caribbean regions of Colombia and Venezuela. Found closed to the beach, mangrove and tropical dry forest (Fig. 1a).

\section{Calanthea Miers.}

2.1. Calanthea pulcherrima (Jacq.) Miers., Proc. Roy. Hort. Soc. London 4: 161. 1864. Capparis pulcherrima Enum. Syst. P1. 24. 1760.

Treelets or shrubs, 1-5 m tall; stem glabrous or stellate in new or terminal branch. Leaves simple, alternated and arranged spirally; petiole angular and ventrally canaliculated, glabrous or stellate on new branch, of similar length, 7-9 $\mathrm{mm}$ long. Blades coriaceous, oblanceolate, ovate to elliptic 7-9.5 × 3-3.5 cm, attenuate, obtuse to rounded at base, obtuse, rounded to emarginate at apex, margin undulate, glabrous mainly or slightly stellate at the base of juvenile leaves on new branch; 10-15 pairs of secondary veins. Racemes terminal, with 8-12 flowers; rachis angular, densely stellate throughout. Flowers actinomorphic and hermaphroditic; subtended by small triangular bracts, densely stellate throughout; pedicel 11 mm long, angular, densely stellate-puberulent. Aestivation of calyx imbricate, sepals oblanceolate, 10-12 $\times 2 \mathrm{~mm}$, acute at apex, stellate at the both sides. Floral nectaries scales deltoid and stellate. Petals imbricate, oblongoid, white or pink, $18 \times 6$ $\mathrm{mm}$, acute at apex, inner side densely stellate and tomentosus in the outside. Stamens 8, filaments exsert, $35 \mathrm{~mm}$ long, glabrous; anthers basifixed, 6 $\mathrm{mm}$ long. Gynophore exserts $21.5 \mathrm{~mm}$ long, densely stellate throughout; ovary oblongoid, 1.3-5.6 mm long, densely stellate throughout; stigma sessile and capitate. Fruits amphisarca, globoid to ovoid, 15-20 cm long; seeds 10-25, ellipsoidal.

Selected specimens examined: COLOMBIA. ATLÁNTICO: entre Leña y Candelaria, 11.I.1941, $A$. Dugand 2785 (COL).

Endemic to the Caribbean region of Colombia. Found close to the beach, mangrove and tropical dry forest (Fig. 1a).

\section{Capparidastrum (DC.) Hutch.}

Trees or shrubs; stem glabrous or less common simple short pilose, with tuberculous lenticels ovoid to elongate. Leaves simple, subtended by pairs of triangular stipules, alternate and arranged spirally; petioles of unequal length with pulvinus at base, apex or both tips, terete or semiterete. Blade coriaceous, membranaceous to papyraceous, acute to acuminate at apex, acute to rounded, cordate even unequal at base, margin entire to slightly undulate, glabrous to slightly pilose. Racemes terminal or subterminal racemes, mainly glabrous. Aestivation of calyx imbricate, valvate; sepals free. Flowers actinomorphic and hermaphroditic. Floral nectaries fleshy, scales deltoid-ovoid and glabrous. Petals twice the size of sepals. Stamens exserts, twice the size of petals. Gynophore exsert, larger than the stamens. Fruits pepo or amphisarca, seeds variable in number, ovoid, ellipsoidal to reniform.

\section{Identification key to the species of Capparidastrum}

1. Blades glabrous throughout

2. Stem with tuberculous lenticels, blades coriaceous to membranaceous, and margin mainly entire

to slightly undulate.

3. Plants no leafy, petals glabrous outside................................................................ 4

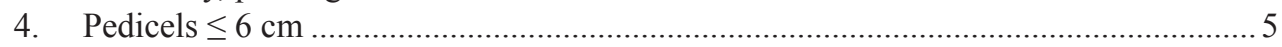

5. Stamens $\leq 35 \mathrm{~mm}$ long, gynophore $\leq 40 \mathrm{~mm}$ long .................................... 6 
6. Anther basifixed and fruits capsular. 3.8. Capparidastrum sola

6'. Anther dorsifixed and fruits in pepo 3.6. Capparidastrum osmanthum

5'. Stamens $\geq 40 \mathrm{~mm}$ long, gynophore $\geq 65 \mathrm{~mm}$ long

3.4. Capparidastrum macrophyllum

4'. Pedicels $\geq 8 \mathrm{~cm}$ long.....

3.5. Capparidastrum megalospermum

3'. Plants leafy, petals pubescent outside

3.2. Capparidastrum frondosum

2'. Stem without tuberculous lenticels, blades chartaceous and, margin strongly undulate

3.7. Capparidastrum pachaca

1'. Blades short pilose beneath

7. Petals $\leq 16 \mathrm{~mm}$ long, stamens $\leq 40 \mathrm{~mm}$ long and gynophore $\leq 90 \mathrm{~mm}$ long....

3.1. Capparidastrum cuatrecasanum

7'. Petals $\geq 20 \mathrm{~mm}$ long, stamens $\geq 30 \mathrm{~mm}$ long and gynophore $\geq 100 \mathrm{~mm}$ long........

3.3. Capparidastrum grandiflorum

3.1. Capparidastrum cuatrecasanum (Dugand) Cornejo \& Iltis, Harvard Pap. Bot.

13(2): 233. 2008. Capparis cuatrecasana Dugand, Caldasia 1(2): 41. 1941.

Trees, $5 \mathrm{~m}$ tall; stem glabrous or pilose on new or terminal branch, densely tuberculous elongate lenticeled. Petiole terete, densely short pilose, 1-12 cm long; pulvinus at both tips. Blades coriaceous, ovate-elliptic, 5-22 × 6-10 $\mathrm{cm}$, acute at base, acute at apex; margin entire, glabrous above and short-pilose beneath; 10-12 pairs of secondary veins. Racemes terminal or subterminal, with 12-15 flowers; rachis terete, densely short pilose. Flowers subtended by triangular bracts; pedicel terete, 20-40 mm long, densely short pilose throughout. Sepals ovate to deltoid, $6 \times 5.5 \mathrm{~mm}$, rounded at apex, short pilose in both sides. Floral nectaries scales glabrous. Petals free, oblong, 9-16 × 6-9 mm, rounded at apex, glabrous. Stamens 20-30, filaments $35 \mathrm{~mm}$ long, glabrous; anthers dorsifixed, 2.5 $\mathrm{mm}$ long. Gynophore $70 \mathrm{~mm}$ long, glabrous; ovary ellipsoidal, $3 \times 1.5 \mathrm{~mm}$, glabrous; stigma sessile, truncate. Fruits pepos, ovoid, $3.5 \times 2-7$ $\mathrm{cm}$, exocarp smooth; seeds $15-30$, reniform to ellipsoidal.

Selected specimens examined: COLOMBIA. CUNDINAMARCA: San Antonio de Tequendama, 10.III.1940, J. Cuatrecasas 8275 (COL). SANTANDER: Matanza, mina La Viborita, $7^{\circ} 15^{\prime} 34.66^{\prime} \mathrm{N}, 7^{\circ}$ 3'36.67'W, 12.XII. 2014, J. Vega 48 (CDMB).

Endemic to the northeast of Colombia, over the East Andean cordillera. Found in wet Andean forest (Fig. 1a).

3.2. Capparidastrum frondosum (Jacq.) Cornejo \& Iltis, Harvard Pap. Bot. 13: 232. 2008. Capparis frondosa Jacq., Enum. Syst. Pl. 24.
1760. Pleuteron frondosa (Jacq.) Raf., Sylva Tellur. 109. 1838. Uterveria frondosa (Jacq.) Bertol., Horti. Bonon. Pl. Nov. 2: 8-9. 1839.

Small trees, shrubs or scandent vines, $6 \mathrm{~m}$ tall; stem glabrous, densely covered by tuberculous elongate lenticels. Petiole terete or angular, glabrous, $0.5-4.5 \mathrm{~cm}$ long; pulvinus at both tips. Blades coriaceous, elliptic to oblanceolate, $8-22 \times 3.8-12.1 \mathrm{~cm}$, cordate to slightly attenuate at base, acute, obtuse, retuse and acuminate at apex; margin entire to undulate, glabrous; 7-10 pairs of secondary nerves. Corymbs racemes terminal, with 4-6 flowers; rachis terete and glabrous. Flowers subtended by small triangular bracts; pedicel terete, $8-11 \mathrm{~mm}$ long, glabrous. Sepals fused at the base, oval to orbicular, $0.8-1.5 \times 1.5 \mathrm{~mm}$, rounded at apex, glabrous on the inner side and simple hairs on the outside. Floral nectaries scales deltoid and glabrous. Petals white-greenish even purple at the base, ovate to orbicular, $8-10 \times 6 \mathrm{~mm}$, rounded at apex, glabrous. Stamens 27-60, filaments 14-15 mm long, glabrous; anthers dorsifixed, 1 $\mathrm{mm}$ long. Gynophore 15-20 mm long, glabrous; ovary elongates $4-5 \mathrm{~mm}$ long, glabrous; stigma sessile, truncate. Fruits capsular, cylindric to oblong, 20-40 × $8 \mathrm{~mm}$, exocarp smooth; seeds $10-20$, ovate to reniform.

Selected specimens examined: COLOMBIA. ARAUCA: Arauca, Mata de Monte, 6 ${ }^{\circ} 48^{\prime} 41.09^{\prime \prime} \mathrm{N}$, 7059'31.57'W, 10.III.2013, F. Mijares 574 (COL, FMB). CASANARE: Paz de Ariporo, La Hermosa 5'36'23.8”'N, 70¹5'35.7'”, 31.X.2004, J. RamirezArango 9123 (FMB). SUCRE: Colosó, Primates, 17.VI.1987, A. Cogollo 2418 (JAUM).

Distributed from Mexico to Peru. Found in tropical dry forest and humid forest, savannah and disturbed forest (Fig. 1b). 
3.3. Capparidastrum grandiflorum Cornejo \& Iltis, Harvard Pap. Bot. 15(1): 155. 2010.

Trees, 7-20 m tall; stem glabrous and densely short pilose at the new branch, tuberculous elongate, orbicular to oblong lenticeled. Petiole terete, glabrous or slightly short pilose, $2-17 \mathrm{~cm}$ long; pulvinus at both tips. Blades chartaceous to coriaceous, ovate to elliptic, 7-31 × 3.5-16 cm, obtuse, rounded at base, apiculate, obtuse and acuminate at apex; margin entire, glabrous above
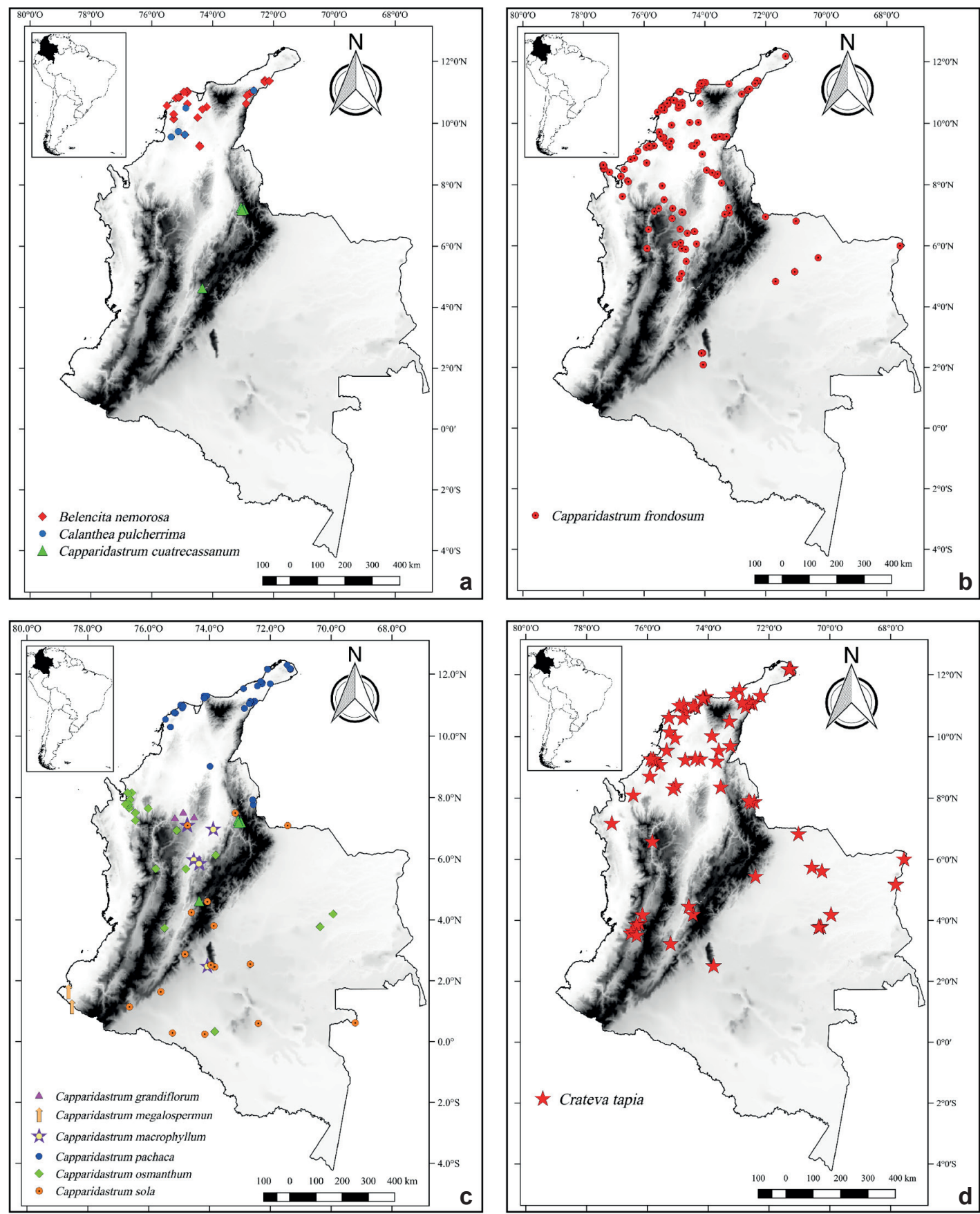

Figure 1 - Distribution map of species of Capparaceae to the flora of Colombia - a. B. nemorosa, C. pulcherrima and C. cuatrecassanum; b. C. frondosum; c. C. grandiflorum, C. megalospermun, C. macrophyllum, C. pachaca, C, osmanthun and C. sola; d. C. tapia. 
and short pilose beneath; 7-8 pairs of secondary veins. Corymbs racemes terminal, with $2-14$ flowers; rachis terete and short-pilose. Flowers subtended by pairs of small triangular bracts, densely short pilose throughout; pedicel terete, 30-70 mm long, densely short pilose throughout. Sepals oval to deltoid, 2-6 $\times 2-6 \mathrm{~mm}$, rounded at apex, glabrous at inner side and short pilose on the outside. Floral nectaries, orbicular and glabrous. Petals imbricate, oblong, white, 20-24 $\times$ 9-13 mm, rounded at apex, glabrous. Stamens $33-50$, filaments $11-13 \mathrm{~cm}$ long, glabrous; anthers dorsifixed, 2-5 mm long. Gynophore exsert, glabrous, $15-20 \mathrm{~cm}$ long; ovary ovate to elliptic, 4-7 mm long, short pilose; stigma sessile, truncate. Fruits pepo pedunculate, ovate, elliptic to oblong 8-10 × 4-7 mm, exocarp smooth; seeds 15-30, ellipsoidal to globoids.

Selected specimens examined: COLOMBIA. ANTIOQUIA: Vía Providencia-Toma de Algibes, a lo largo del Río Anorí, 7¹8’28.02”N, 758'14.02”W, 12.XII. 1987, $R$. Callejas 4567 (HUA).

Endemic to the northwestern foothill of the Central cordillera of Colombia. Found at wet and very wet Andean forest (Fig. 1c).

\subsection{Capparidastrum macrophyllum (Kunth)} Hutch., Gen. Fl. P1. 2: 310. 1967. Capparis macrophylla Kunth., Nov. Gen. Sp. (quarto ed.) 5: 91. 1821. Capparis iltisiana T. Ruiz, Ernstia 36: 1-2. 1986. Capparis magnifica Gilg ex Ule., Bot. Jahrb. Syst. 40: 140. 1907.

Trees or shrubs, $20 \mathrm{~m}$ tall, with scandent branches present; glabrous, densely tuberculous elongate lenticeled. Petiole 4-20 cm long, terete, glabrous, pulvinus present on both tips. Blades coriaceous to papyraceous, ovate to elliptic 10-31 $\times$ $10-25 \mathrm{~cm}$, base obtuse, rounded, apex acuminate to acute; margin entire to slightly undulated, glabrous; 9-13 pairs of secondary veins. Inflorescences terminal, corymbs racemes with 6-16 flowers; rachis terete and glabrous. Flowers subtended by small ovate bracts, glabrous; pedicel angular 20-60 mm long, glabrous. Sepals deltoid 2-7.5 $\times 2-6 \mathrm{~mm}$, rounded at the apex, glabrous. Floral nectaries orbicular and glabrous. Petals imbricate, white or cream, oval to oblong 20-35 × 15-20 $\mathrm{mm}$, apex rounded, glabrous. Stamens 80-130, filaments very exsert $65-110 \mathrm{~mm}$ long, glabrous; anthers dorsifixed, 10-12 mm long. Gynophore very exsert, 70-120 mm long, glabrous; ovary ovate to elliptic 9-10 × 3-3.3 mm, glabrous; stigma sessile and truncate. Fruits capsular, oblongs to elongate 5-13.5 × 3.5-7 mm, exocarpo smooth; seeds 25-65, ellipsoidal to globoids.

Selected specimens examined: COLOMBIA. META: La Macarena, PNN Tiniguas, Centro de Investigaciones Primatológicas Macarena, 1985, H. Tasuyuki 214 (FMB).

Distributed in Venezuelan and Colombian Andes, lowlands of Ecuador, Peru and Brazil. It is found in wet to very wet Andean forest and lowland forest (Fig. 1c).

3.5. Capparidastrum megalospermum Cornejo \& Iltis, Harvard Pap. Bot. 15(1): 159, f. 2, 4. 2010.

Trees, $15 \mathrm{~m}$ tall; stem glabrous, tuberculous lenticels oblong-elliptic to suborbicular present. Stipules unknown; petiole 2-37 cm long, (sub) terete, glabrous, pulvinus present on both tips. Blades chartaceous to coriaceous, ellipticlanceolated to elliptic-obovate $20-40 \times 7-20 \mathrm{~cm}$, base obtuse to cuneate, apex acuminate to acute; margin entire, glabrous; 8-13 pairs of secondary veins. Inflorescences terminal, racemes with 10 flowers, rachis terete and glabrous; pedicels $80 \mathrm{~mm}$ long, glabrous. Sepals imbricate, deltoid $6 \times 4 \mathrm{~mm}$. Fruits in pepo, pendulous capsulate, ellipsoid $15 \times$ $8 \mathrm{~mm}$, exocarp smooth; seeds 10-25 ellipsoidal. Selected specimens examined: COLOMBIA. NARIÑO: Costa del Pacífico, Herrera, 27-29.VI.1953, Idrobo \& Weber 1420-E (COL).

Distributed from southwestern of Colombia to Equator. Found at wet to very wet Andean forest and lowland forest (Fig. 1c).

3.6. Capparidastrum osmanthum (Diels) Cornejo \& Iltis, Harvard Pap. Bot. 13(2): 234. 2008. Capparis osmantha Diels., Notizbl. Bot. Gart. Berlin-Dahlem 14: 332. 1939. Capparis guaguaensis Steyerm., Fieldiana, Bot. 28(1): 238. 1951. Capparis macrophylla Kunth., Nov. Gen. Sp. (quarto ed.) 5: 91. 1821.

Small trees or shrubs, $5 \mathrm{~m}$ tall; glabrous throughout, with tuberculous lenticels elongates, in mature stem lenticels inconspicuous. Petioles terete, glabrous, 5-12 cm long; pulvinus at both tips. Blades membranous to papyraceous, elliptic to ovate-elliptic, $8-17 \times 4.5-8.5 \mathrm{~cm}$, acute at base, acuminate to acute at apex; margin entire; 8-18 pairs of secondary veins. Corymbs racemes terminal, with 5-15 flowers; rachis (sub)terete and glabrous. Flowers subtended by pairs of triangular and deciduous bracts; pedicels terete, $20-35 \mathrm{~mm}$ long, glabrous or short pilose. Sepals fused at the base, ovate to deltoid, $2 \times 1-2 \mathrm{~mm}$, acute to rounded at apex. Floral nectaries orbicular and 
glabrous, after senescence the glands are bigger than sepals. Petals frees, elliptic-oblongate to ovate, white, $8-16 \times 10 \mathrm{~mm}$, rounded to obtuse at apex, glabrous. Stamens 20-30, filaments 25-35 mm long, glabrous; anthers dorsifixed, 4 mm long. Gynophore 30-40 mm long, glabrous; ovary ovoid $4 \times 2 \mathrm{~mm}$; stigma sessile, capitate. Fruits pepo, pedunculate, elliptic to oblong 3-5 × 3-4 cm; seeds 30-40, elliptic to reniform.

Selected specimens examined: COLOMBIA. AMAZONAS: Puerto Nariño, trapecio amazónico, J.M. Duque-Jaramillo 2149 (COL). CAQUETÁ: Río Caguán, 20.VI.1953, R. Romero-Castañeda 4022 (COL).

Distributed in Colombia, Venezuela, east of Ecuador, Brazil and Peru. Found in wet to very wet forest (Fig. 1c).

3.7. Capparidastrum pachaca (Kunth) Hutch., Gen. F1. P1. 2: 310. 1967. Capparis pachaca Kunth., Nov. Gen. Sp. (quarto ed.) 5: 93. 1821.

Small trees or shrubs, $10 \mathrm{~m}$ tall; stem glabrous throughout; tuberculous lenticels present in young stem, however when mature inconspicuous. Petioles of similar length, 3-12 cm long, (sub)terete, ventrally canaliculate, glabrous; pulvinus inconspicuous or absents. Blades coriaceous and rigid, oblongate-ovate to oblanceolate, $7-16 \times 4-8 \mathrm{~cm}$, obtuse, cuneate to rounded at base, obtuse at apex; margin strongly undulate, 7-10 pairs of secondary veins. Racemes terminal or axillar, with 9-16 flowers; rachis terete and glabrous. Flowers subtended by pairs of linear bracts; pedicels terete, 8-22 mm long, glabrous or short pilose in senescence. Sepals fused, lobes deltoid, 1-2 × 2-3 mm, rounded at apex. Floral nectaries scales deltoid and glabrous. Petals imbricate, ovate to elliptic, white or cream, 9-12 $\times$ 6-9 mm, rounded to obtuse at apex, glabrous. Stamens 40-63, filaments exserts, 20-40 mm long, glabrous, anthers dorsifixed, $2.5 \mathrm{~mm}$ long. Gynophore exsert, 20-50 mm long, glabrous; ovary elliptic to ovoid 1.5-5 × 1-1.2 mm; stigma sessile, capitate. Fruits amphisarca globoids, $2.8-3.8 \times 2.3-3.5 \mathrm{~cm}$, exocarp slightly verrucose; seeds 14-17, reniform.

Selected specimens examined: COLOMBIA. ATLÁNTICO: Cerro El Palomar, 18.VIII.2011, C. Castellanos 546 (COL). NORTE DE SANTANDER: San José de Cúcuta, San Pedro, carretera entre la mina El Chipio y el río Pamplonita, 746'2.1'N, 72³3'34.2'W, 30.I.2009, J. Infante 414 (COL).

Distributed from Mesoamerica to Caribbean region of Colombia. Found in tropical dry forest, disturbed forest and slightly wet forest (Fig. 1c).
3.8. Capparidastrum sola (J.F. Macbr.) Cornejo \& Iltis, Harvard Pap. Bot. 11(1): 17. 2006. Capparis sola J.F. Macbr., Candollea 5: 359. 1934. Capparis acutifolia J.F. Macbr., Candollea 5: 358. 1934.

Trees or shrubs, 3-10 m tall; stem glabrous throughout; tuberculous orbicular lenticeled. Petioles terete, glabrous, 5-16 cm long; pulvinus at both tips. Blades coriaceous, elliptic, 15-22 $\times$ 6-9 cm, cuneate to cordate at base, acute at apex; margin entire; 10-12 pairs of secondary veins. Racemes terminal or axillar, elongate, with 11-28 flowers; rachis angular and glabrous. Flowers subtended by pairs of triangular bracts; pedicels terete, 1.2-2.5 mm long and glabrous. Sepals fused at base, orbicular to deltoid, $1 \times 1 \mathrm{~mm}$, rounded to obtuse at apex and glabrous. Floral nectaries orbicular and glabrous. Petals imbricate, oblong to ovate, white or cream, 7-8 $\times 5 \mathrm{~mm}$, obtuse at apex, glabrous. Stamens 100- $\infty$, filaments 10-30 $\mathrm{mm}$ long, glabrous; anthers basifixed, $1.3 \mathrm{~mm}$ long. Gynophore $30 \mathrm{~mm}$ long, glabrous; ovary globoid 2 $\times 1.5 \mathrm{~mm}$; stigma sessile, capitate. Fruits capsular, cylindric-oblong, torulous 10-50 × 20-30 cm, exocarp smooth; seeds 7-10 ovate to reniform.

Selected specimens examined: COLOMBIA. AMAZONAS: Solano, Araracuara, Río Caquetá, 07.VIII.1977, J.M. Idrobo 8946 (COL). GUAVIARE: San José de Guaviare, PNN Nukak, 02.II.1996, M.P. Cordoba et al. 1866, 1872 (FMB).

Distributed in the Colombian Amazonia, Venezuela and Brazil. Found in wet to very wet forest (Fig. 1c).

\section{Crateva L.}

4.1. Crateva tapia L., Sp. P1. 1: 444. 1753. Capparis radiatiflora (DC.) Ruiz \& Pav. ex E. Álvarez L., Anales Inst. Bot. Cavanilles 16: 387, t. 433. 1958. Capparis ternata Tafalla., F1. Huayaquilensis 1: 161. 1989. Cleome arborea Schrad., Nov. Gen. Sp. (quarto ed.) 2(72): 707. 1821. Colicodendron obliquifolium Turcz., Bull. Soc. Imp. Naturalistes Moscou 27(3): 328. 1854. Crateva acuminata DC., Prod. 1: 243. 1824. Crateva apetala Urb., Symb. Antill. 7: 508. 1913. Crateva bahiana Ule., Bot. Jahrb. Syst. 42(2): 202. 1908. Crateva benthamii Eichler., F1. Bras. 13(1): 265-266, t. 59. 1865. Crateva benthamii var. leptopetala Eichler., Fl. Bras. 13(1): 265-266. 1865. Crateva coriacea Herzog., Repert. Spec. Nov. Regni Veg. 7(134/136): 52. 1909. Crateva gynandra L., Sp. Pl. 1: 636-637. 1762. Crateva radiatiflora DC., Prod. 1: 243. 1824. Crateva tapia var. glauca (Lundell) Standl. \& Steyerm., Publ. Field Mus. Nat. Hist., 
Bot. Ser. 23(2): 55. 1944. Crateva tapioides DC., Prod. 1: 243. 1824. Capparis ternata Tafalla, Fl. Huayaquilensis 1: 161. 1989.

Trees or shrubs, 10-30 m tall; glabrous throughout, with tuberculous lenticels elongated, very abundant. Leaves palmately-compound, 3-foliolate, alternate and arranged in spiral, subtended by pairs of small triangular stipules; petioles angular, glabrous, unequal in size 5-20 $\mathrm{cm}$ long, very long. Leaflet membranaceous, elliptic ovate, ovate-elliptic, $8-20 \times 2-10 \mathrm{~cm}$; central leaflet: petiolule $4-10 \mathrm{~mm}$ long, base cuneate to acute; apex acuminate, acute to round; margin entire. Corymbose racemes terminal, with 6-20 flowers; rachis angular, ventrally canaliculate and glabrous. Flowers zygomorphic and hermaphroditic, perigynous, subtended by pairs of linear to lanceolate bracts; pedicels terete, 20-50 mm long, glabrous. Aestivation of calix decussate; sepals lanceolate, oblong to ovate, 5-15 $\times 1-3 \mathrm{~mm}$; rounded at apex. Floral nectaries glabrous. Petals decussate, lanceolate, oblong, unguiculate, to spathulate; $8-45 \times 2-13 \mathrm{~mm}$; base of petals of 4-10 mm long; rounded at apex. Stamens 14-20; filaments very exserts, $8-50 \mathrm{~mm}$ long, glabrous; anthers basifixed, $3.5 \mathrm{~mm}$ long. Gynophore 10-60 mm long, glabrous; ovary ovoid, 4-7 ×3-4 mm; stigma discoid, subsessile, capitate. Fruits amphisarca, globoids, $3-8 \mathrm{~cm}$ diam; exocarp smooth, granulose or with lenticels; seeds 7-20, reniform.
Selected specimens examined: COLOMBIA. AMAZONAS: Parque Nacional Natural Amacayacú, 06.VIII.1988, M. Amaya 49 (COL). ATLÁNTICO: Barranquilla, zona industrial en cercanías al Río Magdalena, $11^{\circ} 1^{\prime} 43.23$ 'N, 7448'51.41'W, 09.III.2013, J.M. Vélez-Puerta et al. 4365, 4373 (MEDEL). CUNDINAMARCA: Pandi, vereda El Yarumo, puente sobre el Río Negro en el cruce con el río Sumapaz, 23.XI.1998, K. Poveda 29 (COL).

Distributed from Mexico to Argentina. Found in a wet to very wet forest, lowland and tropical dry forest (Fig. 1d).

\section{Cynophalla (DC.) J. Presl.}

Trees or shrubs; stem mainly glabrous or short pilose on new branch. Extrafloral supraxillary nectaries present in terminal or new branch. Leaves simple, alternate and terminal branch with distichous and zigzag phyllotaxy, subtended by a pair of small triangular stipules; petioles of similar length, pulvinus absent, sub(sessile). Corymbose racemes or racemes terminal. Flowers actinomorphic and hermaphroditic, some flowers are suppressed or are glands located in each angle. Aestivation of calix imbricate, sepals biseriate, inner whorls bigger than the outer ones. Petals imbricate, bigger than sepals, apex orbicular. Petals with aestivation imbricate. Stamens numerous, exserts, mainly glabrous, anther dorsifixed. Gynophore exsert; ovary mainly cylindric. Fruits capsular, torulous or no-torulous; seed variable in number.

\section{Identification key to the species of Cynophalla}

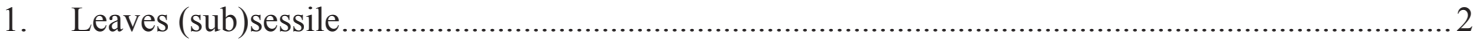

2. Stem and blades short pilose. Fruits with smooth exocarp ...................... 5.6. Cynophalla sessilis

2'. Stem and blades glabrous. Fruit with verrucose exocarp..................... 5.7. Cynophalla verrucosa

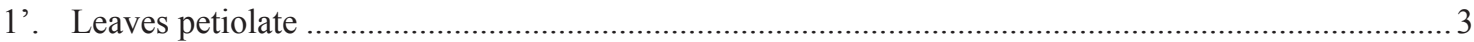

3. Blades linear................................................................................ 5.4. Cynophalla linearis

3'. Blades ovate, oblong, spathulate, ellipsoidal, oblanceolate or hastate ...................................... 4

4. Blades with margin revolute and sepals fused at base .............. 5.1. Cynophalla amplissima

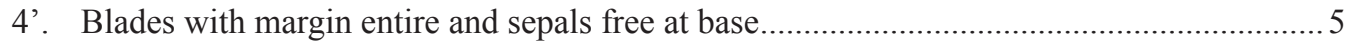

5. Blades retuse at the apex. Flowers with more than 100 stamens

5.2. Cynophalla flexuosa

5'. Blades acute to acuminate at the apex. Flowers with less of 90 stamens

6. Blades membranaceous and acuminate at the apex. Fruits torulous and exocarp smooth.

5.5. Cynophalla polyantha

6'. Blades coriaceous to chartaceous and acute at the apex. Fruits bacciform and exocarp verrucose 5.3. Cynophalla hastata 
5.1. Cynophalla amplissima (Lam.) Iltis \& Cornejo, Rodriguésia 61(1): 154. 2010. Capparis amplissima Lam., Encycl. 1: 607. 1783. Capparis nitida Ruiz \& Pav. ex DC., Prod. 1: 252. 1824. Capparis pendula Triana \& Planch., Ann. Sci. Nat., Bot., sér. 4, 17: 76-77. 1862.

Trees or shrubs, 10-20 m tall; stem glabrous or short pilose at the new branch. Petioles sub(terete), ventrally canaliculated, angular, 5-20 cm long, glabrous. Blades chartaceous to membranaceous, elliptic, oblanceolate, elliptic-oblongate, 5-18 $\times$ 3-8 cm, subcordate, obtuse and rounded at base, acuminate to obtuse at apex; margin revolute, glabrous; 9-12 pairs of secondary veins. Racemes terminal or axillar, with 6-15 flowers; rachis terete and glabrous. Pedicels terete, short-pilose or glabrous, $10-15 \mathrm{~mm}$ long. Sepals fused at the base, rounded at apex: inner series 10-12 × 12-15 $\mathrm{mm}$ and the outer 6-8 $\times 11-12 \mathrm{~mm}$ both glabrous. Petals oblong-ovate, withe, $20-30 \times 10-12 \mathrm{~mm}$, rounded to obtuse at apex. Stamens $80-\infty$, filaments $50-80 \mathrm{~mm}$ long, with glandular trichomes at the base; anthers dorsifixed, 4-5 mm long. Gynophore 60-90 mm long, glabrous; ovary oblongoid 2-4 $\times$ $0.7-1.1 \mathrm{~mm}$, glabrous; stigma sessile, truncated. Fruits pendular, oblongoid to ovoid, 4-8 $83-5 \mathrm{~cm}$, exocarp smooth; seeds 12-17, elliptic to oblongoid. Selected specimens examined: COLOMBIA. ANTIOQUIA: San Luis de Cocorná, 19.XI.1963, $R$. Romero-Castañeda 10101 (UTMC). SUCRE: Colosó, Primates, 9³1'37.43”'N, 75²1'54.18'W, 17.VI.1987, A. Cogollo 2412 (JAUM). VALLE DEL CAUCA: Vía Loboguerrero-Dagua, $03^{\circ} 42^{\prime} 05.6^{\prime}$ 'N, 76 $40^{\prime} 53.7^{\prime}$ 'W, 2.XI.2013, N. Castaño 271 (TULV).

Distributed from Mesoamerica to Colombia, Venezuela, Bolivia, Brazil, Puerto Rico and the Hispaniola. Found in wet forest, Andean and tropical dry forest (Fig. 2a).

5.2. Cynophalla flexuosa (L.) J. Presl, Přir. Rostlin 2: 275. 1825. Morisonia flexuosa L., Pl. Jamaic. Pug. 14. 1759. Capparis baducca Sessé \& Moc., Fl. Mexic. (ed. 2). 129. 1894. Capparis brevisiliqua DC., Prodr. 1: 251. 1824. Capparis cynophallophora var. biflora Bello, Anales Soc. Esp. Hist. Nat. 10: 237. 1881. Capparis cynophallophora var. elliptica Bello, Anales Soc. Esp. Hist. Nat. 10: 237. 1881. Capparis diversifolia Sessé \& Moc., Fl. Mexic. 130. 1894. Capparis flexuosa (L.) L., Sp. Pl. (ed.2) 1: 722.1762.

Small trees, shrubs or vines, $10 \mathrm{~m}$ tall; stem glabrous throughout. Petioles angular, terete, ventrally canaliculated, 5-10 $\mathrm{mm}$ long, glabrous.
Blades coriaceous and rigid, elliptic, oblong to oblongs-deltoids, 3-10 $\times 2-7 \mathrm{~cm}$, cuneate, obtuse and rounded to subcordate at base, retuse to slightly obtuse at apex; margin entire, glabrous; 8-10 pairs of secondary veins. Racemes terminal, with 6-8 flowers; rachis terete and glabrous. Pedicels terete, glabrous or short-pilose, 10-20 mm long. Sepals imbricate or fused at the base, rounded to obtuse at apex: inner series 7-8 $\times 8-10 \mathrm{~mm}$ and outer series 5-6 $\times 4-5 \mathrm{~mm}$. Floral nectaries scales deltoids and glabrous. Petals oblongs, white or pink, $15-30 \times$ 10-20 mm, rounded to obtuse at apex. Stamens $100-\infty$, filaments $40-80 \mathrm{~mm}$ long, with glandular trichomes at the base; anthers basifixed, 3-5 mm long. Gynophore 20-50 mm long, glabrous; ovary oblongoid to cylindric 4-7 $\times 0.5-1 \mathrm{~mm}$, glabrous; stigma sessile, truncate. Fruits pendular, linear to cylindric, torulous, 6-15 $\mathrm{cm} \times 4-8 \mathrm{~mm}$, exocarp smooth; seeds 8-22, oblongoid.

Selected specimens examined: COLOMBIA. ARCHIPIÉLAGO DE SAN ANDRÉS, PROVIDENCIA Y SANTA CATALINA: North cliff, 26.XII.1990, $P$. Lowy 304 (COL). BOLÍVAR. Zambrano, Finca Forestal Monterrey, 9³8'20.20'N, 7454'31.76'W, 1.VIII.1996, H. Mendoza 1468 (COL, FMB). SANTANDER: Piedecuesta, sector Cabrera, $7^{\circ} 5^{\prime} 12.16^{\prime \prime} \mathrm{N}, 7^{\circ} 5^{\prime} 0.20^{\prime \prime} \mathrm{W}$, 11.XII. 2002, L.A. Pinto 14 (CDMB).

Distributed from Central America to Colombia and Venezuela. Found in close proximity to the beach and tropical dry forest (Fig. 2a).

5.3. Cynophalla hastata (Jacq.) J. Presl., Přir. Rostlin 2: 275. 1825. Capparis hastata Jacq., Enum. Syst. P1. 23. 1760. Capparis flexuosa fo. hastata (Jacq.) Dugand, Caldasia 2: 51. 1941. Pleuteron hastata (Jacq.) Raf., Sylva Tellur. 109. 1838. Capparis coccolobifolia Mart. ex Eichler., Fl bras. 13(1): 284. 1865. Capparis collina J.R. Johnst., Proc. Amer. Acad. Arts 40: 686. 1905.

Small trees or shrubs, $10 \mathrm{~m}$ tall; stem glabrous or short pilose over new branch. Petioles 5-10 $\mathrm{mm}$ long, terete, ventrally canaliculate, angular, glabrous. Blades coriaceous and rigid, elliptic, oblongs to oblongs-deltoids, 3-10 × 2-7 cm, base cuneate, obtuse and rounded to subcordate; retuse to slightly obtuse at the apex, margin entire, glabrous, 8-10 pairs of secondary veins. Inflorescences terminal, corymbose racemes with 7-16 flowers; rachis terete and glabrous. Pedicels 7-12 mm long, terete, glabrous or short-pilose. Sepals imbricate or decussate, apex rounded to retuse: inner series 4-6 $\times 7-8 \mathrm{~mm}$ and outer 3-5 $\times$ 6-7 $\mathrm{mm}$, both short pilose outside or glabrous. 

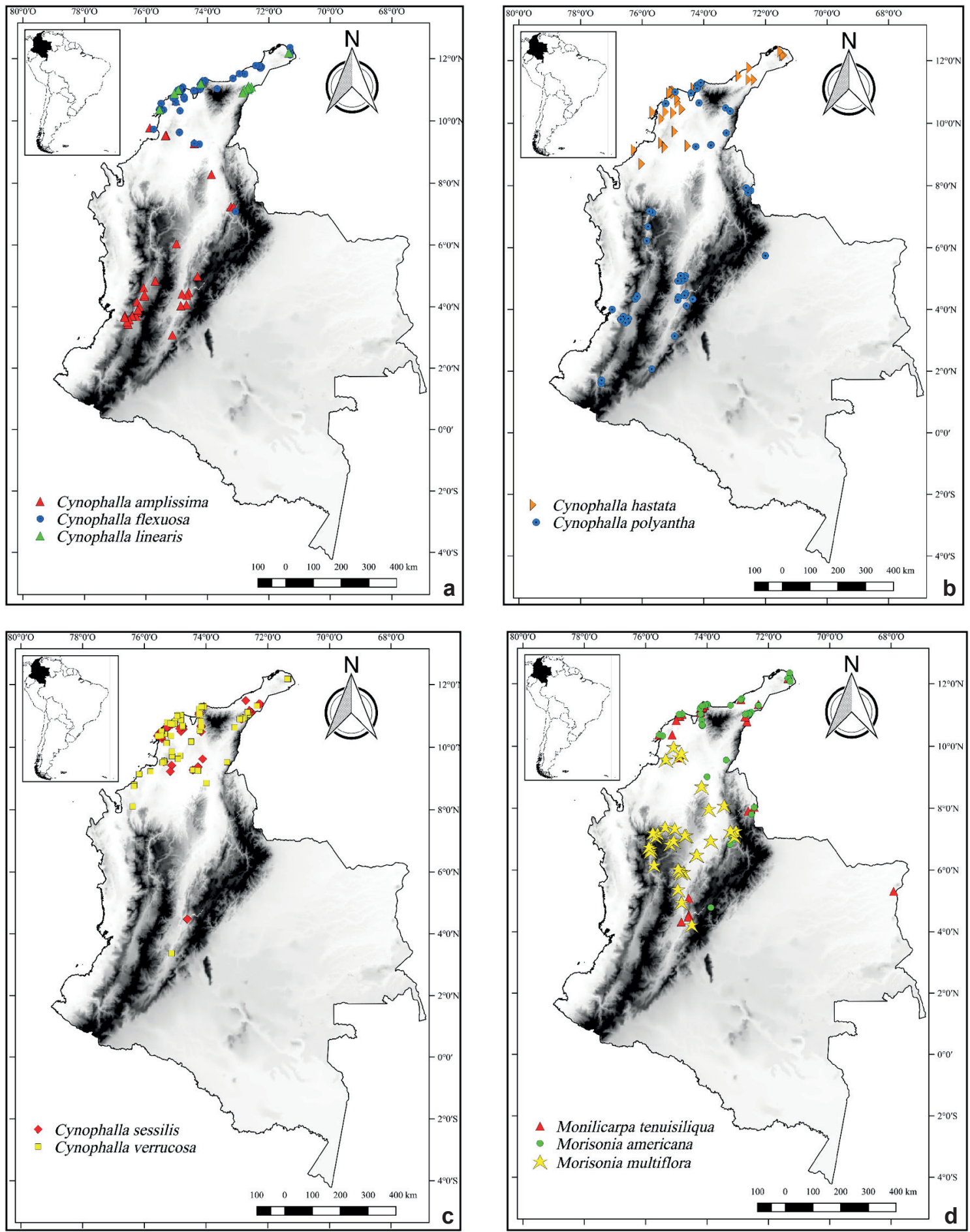

Figure 2 - Distribution map of species of Capparaceae to the flora of Colombia-a. C. amplissima, C. flexuosa and C. linearis; b. C. hastata, C. linearis; c. C. sessilis and C. verrucosa; d. M. tenuisiliqua, M. americana and M. multiflora. 
Floral nectaries, concave and glabrous. Petals greenish, obovate $18-30 \times 11-13 \mathrm{~mm}$; apex rounded to obtuse. Stamens 50-90, filaments 23-44 $\mathrm{mm}$ long, short pilose at the base; anthers basifixed, $4 \mathrm{~mm}$ long. Gynophore exsert, 23-43 mm long, short pilose; ovary oblongoid to cylindric 6-10 $\times$ 1-2 mm, glabrous; stigma sessile and truncate. Fruits bacciform, cylindric, no torulous, 5.5-20 $\times$ $1.1-1.8 \mathrm{~cm}$, exocarp glabrous, slightly verrucose; seeds 8-22, ellipsoidal.

Selected specimens examined: COLOMBIA. BOLÍVAR: Isla de Tierra Bomba, 10²1'41.03'N, 75³3'46.09'W. XIII.1996, H. Mendoza 1795 (FMB). Zambrano, Finca Forestal Monterrey, 15.XIII.1991, S. Zuluaga 172 (FMB). MAGDALENA: San Sebastián, San Valentín, NE de Mompox, 9¹6'39.13'N, 74²5'33.17’'W, 25.II.2000, R. Callejas 12433 (COL, HUA).

Distributed in the north of Colombia, Venezuela and Caribbean Islands. It is found close to beach and tropical dry forest (Fig. 2b).

5.4. Cynophalla linearis (Jacq.) J. Presl., Přir. Rostlin 2: 275. 1825. Capparis linearis Jacq., Enum. Syst. P1. 24: 1760. Pleuteron linearis (Jacq.) Raf., Sylva Tellur. 109. 1838. Uterveria linearis (Jacq.) Horti Bonon., P1. Nov. 2: 10. 1839.

Shrubs or small trees, $6 \mathrm{~m}$ tall; stem glabrous throughout. Petioles terete, 4-6 mm long, ventrally canaliculate, angular and glabrous. Blades coriaceous and rigid, linear 7-17 $\times$ $0.3-1 \mathrm{~cm}$, base obtuse; obtuse at the apex, margin revolute, glabrous, venation with $25-28$ pairs of lateral nerves. Inflorescences terminal or axillar, corymbose racemes with 6-12 flowers; rachis terete and glabrous. Pedicels 7-12 mm long, terete and glabrous. Sepals imbricate or decussate, apex rounded to obtuse, orbicular and biseriate: inner series 5-6 6 3-4 $\mathrm{mm}$ and outer $3 \times 2 \mathrm{~mm}$, both glabrous. Floral nectaries, concave and glabrous. Petals imbricate, white, obovate 8-10 × 8-10 mm, apex rounded to obtuse. Stamens 60-70, filaments 8-10 mm long, glabrous; anthers basifixed $1.5 \mathrm{~mm}$ long. Gynophore inconspicuous or not developed, insert; ovary oblongoid to cylindric 4-6 × 5-10 $\mathrm{mm}$, glabrous; stigma sessile and truncate. Fruits oblong to cylindric, no torulous, 4-6 $61 \mathrm{~cm}$, exocarp smooth; seeds 4-50, ellipsoidal and arranged in two rows.

Selected specimens examined: COLOMBIA. ATLÁNTICO: Juan de Acosta, al lado de la playa de Feru (Santa Verónica), 1052'28.67'N, 754'41.74'W, 10.III.1963, A. Dugand 6216 (COL). LA GUAJIRA: Cerca de Barranca, 1053'34.50”N, 7251'0.31'W, 11.II.1944, O. Haught 3990 (COL).
Distributed in the north of Colombia and Venezuela. It is found close to beach and tropical dry forest (Fig. 2a).

5.5. Cynophalla polyantha (Triana \& Planch.) Cornejo \& Iltis, Harvard Pap. Bot. 13(1): 118-119. 2008. Capparis polyantha Triana \& Planch., Ann. Sci. Nat., Bot., sér. 4, 4 17: 76. 1862. Capparis flexuosa subsp. polyantha (Triana \& Planch.) Iltis., Fl. Venez. Guayana 4: 139. 1998.

Shrubs or small trees, $6 \mathrm{~m}$ tall; stem glabrous throughout. Petioles 7-13 mm long, terete, ventrally canaliculated, angular and glabrous. Blades coriaceous to papyraceous, elliptic to ovate, 9-15 × 3-9 cm, base cuneate, obtuse at the apex, margin entire, glabrous; venation with 8-12 pairs of secondary veins. Inflorescences terminal or axillar, corymbose racemes, with 6-12 flowers; rachis terete and glabrous. Pedicels 5-10 mm long, terete, slightly angular and glabrous. Sepals decussate to imbricate, apex rounded to obtuse, orbicular and biseriate: inner series $10 \times 10 \mathrm{~mm}$ and outer $8 \times$ $8 \mathrm{~mm}$, glabrous. Floral nectaries, concave and glabrous. Petals white, orbicular 8-10 × 8-10 mm, apex rounded to obtuse. Stamens 55-60, filament exsert of $30 \mathrm{~mm}$ long, glabrous; anthers basifixed of $3 \mathrm{~mm}$ long. Gynophore exsert of 40-60 mm long, glabrous; ovary oblongoid to cylindric 7-8 $\times 1$ mm, glabrous; stigma sessile discoid and capitated. Fruits capsular, cylindric, torulous, 5-30 × 0.5-1 $\mathrm{cm}$, exocarp smooth; seeds 4-15, ellipsoidal and arranged in two rows.

Selected specimens examined: COLOMBIA. ARCHIPIÉLAGO DE SAN ANDRÉS, PROVIDENCIA Y SANTACATALINA: Providencia, south hill, Archibolds point, rocky point, Nanchioneal bay, Alligator point, 19-29.XII.1966, J. Torres 347 (COL). ATLÁNTICO: Luruaco, Pendales, Alto de Matamba, $150 \mathrm{~m}, 10^{\circ} 38^{\prime} 00$ '”N, 75¹2'00'”W, 28.XIII.2001, J. Pérez 1793 (JAUM). CASANARE: Pore, vereda Altamira, 544'14.93”N, 720'11.89'W, 01-06.X.2006, Prieto 5054 (FMB). CAUCA: Valle del Patía, $1^{\circ} 5742^{\prime \prime N}, 77^{\circ} 11^{\prime} 58^{\prime}$ W, 06.X.2007, P. Ramírez 20185 (COL). VALLE DEL CAUCA: Bolívar, la Montañuela, 04²0'33.22' N, 76¹3'33.49”'W, 11.XI.2013, Castaño Net al. 312 (TULV).

Distributed mainly in Colombia, Venezuela and Bolivia. It is found at tropical dry forest, wet and Andean forest (Fig. 2b).

5.6. Cynophalla sessilis (Banks. ex DC.) J. Presl, Přir. Rostlin 2: 275. 1825. Capparis sessilis Banks. ex DC., Prod. 1: 249.

Shrubs or small trees mainly erect or flexuous, $4 \mathrm{~m}$ tall; stem pubescent with orbicular 
lenticels. Petioles (sub)sessile and pubescent. Blades coriaceous to papyraceous, elliptic to ovate, $8-10 \times 3-5 \mathrm{~cm}$, base cordate, acute to slightly acuminate at the apex, margin entire, pubescent both, beneath and above, venation with 10-12 pairs secondary veins. Inflorescences terminal or axillar, corymbose racemes with 10-12 flowers, rachis terete and glabrous. Pedicels 5-18 mm long, ventrally canaliculated, terete or slightly angular and pubescent. Sepals imbricate, apex rounded to obtuse, orbicular and biseriate: inner series $5 \times$ $4 \mathrm{~mm}$ and outer $3 \times 4 \mathrm{~mm}$, both glabrous. Floral nectaries, concave and glabrous. Petals imbricate to decussate, cream until greenish, orbicular to ovate $10-13 \times 7-10 \mathrm{~mm}$, apex rounded to obtuse. Stamens 90-100, filament exsert of 20-25 mm long, glabrous; anthers basifixed of $3 \mathrm{~mm}$ long. Gynophore exsert of 18-20 mm long, glabrous; ovary cylindric $4-6 \times 1.2 \mathrm{~mm}$, glabrous; stigma sessile and truncated. Fruits, torulous, cylindric, 8-10 × 0.5-1.2 cm, exocarp smooth; seeds 4-25, ellipsoidal and arranged in two rows.

Selected specimens examined: COLOMBIA. ANTIOQUIA: Anza, km 42 de Bolombolo a Santa Fe de Antioquia, 6 $17^{\prime} 34.07^{\prime}$ N , 7550'50.41''W, 29.XII. 2013, D. Zapata 279 (JAUM). CUNDINAMARCA. Tocaima, Hacienda El Cucharo, 12.X.1943, M.R. Jaramillo 225 (COL). LA GUAJIRA: El Cerrejón, $11^{\circ} 10^{\prime} 1.43$ ”N, 72॰35'39.23"W, 22.VI.2013, C. Sarmiento 500 (COL). SUCRE: Colosó, Primates, 9³1'37.43”N, 75²1'54.18'W, 27.VI.1997, L. Prado 146, 301 (FMB).

Distributed in Colombia and Venezuela. Found in tropical dry forest and disturbed forest (Fig. 2c).

5.7. Cynophalla verrucosa (Jacq.) J., Presl. Přir. Rostlin 2: 275. 1825. Capparis verrucosa Enum. Syst. P1. 23. 1760. Capparis brevipes Benth., Bot. Voy. Sulphur 65. 1844. Capparis laeta Kunth., Nov. Gen. Sp. (quarto ed.) 5: 88. 1821. Capparis palmeri Rose., Contr. U.S. Natl. Herb. 1(9): 301. 1895. Capparis rugulosa Turcz., Bull. Soc. Imp. Naturalistes Mosco 27(2): 326. 1854. Capparis trinitensis Notizbl., Bot. Gart. Berlin-Dahlem 8(71): 25-26. 1921. Uterveria verrucosa (Jacq.) Bertol., Horti Bonon. P1. Nov. 2: 9. 1839.

Small trees, or flexuous shrubs, $4 \mathrm{~m}$ tall; stem glabrous throughout with orbicular lenticels. Petioles (sub)sessile and glabrous. Blades coriaceous elliptic to ovate, $3-13 \times 2-5 \mathrm{~cm}$, base cordate and acute, obtuse to emarginated at the apex, margin revolute, glabrous, venation with 8-10 pairs of lateral nerves. Inflorescences terminal or axillar, corymbose racemes with 4-10 flowers; rachis terete and glabrous. Pedicels 5-15 mm long, ventrally canaliculated, terete and glabrous. Sepals imbricate to decussate, apex rounded to obtuse, orbicular and biseriate: inner series $6 \times$ $5 \mathrm{~mm}$ and outer $4 \times 4 \mathrm{~mm}$, both glabrous. Floral nectaries, concave and glabrous. Petals white cream, orbicular to ovate $12-20 \times 8-12 \mathrm{~mm}$, apex rounded to retuse. Stamens 40-100, filament exsert of 20-30 mm long, glabrous; anthers basifixed of 1.2-2 mm long. Gynophore exsert of 20-30 $\mathrm{mm}$ long, glabrous; ovary oblong $3-7 \times 1.5-2.5$ $\mathrm{mm}$, glabrous; stigma sessile and capitated. Fruits capsular pendulous, cylindric, torulous, 8-10 × 1-3 cm, exocarp verrucose; seeds 10-20, reniform and arranged in two rows.

Selected specimens examined: COLOMBIA. ATLÁNTICO: Piojó, cerro La Vieja, 1044'4.06”N, 756'23.4”W, 11.XII.2015, A. Aguirre 58 (COL). CESAR. La Jagua de Ibirico, La Victoria de San Isidro, Escuela Nueva El Zumbador, 18.III.1996, J. Fernández 13953 (COL).

Distributed from Mexico to the north part of Colombia and Venezuela. Found in tropical dry forest (Fig. 2c).

\section{Monilicarpa Cornejo \& Iltis.}

6.1. Monilicarpa tenuisiliqua (Jacq.) Cornejo \& Iltis, J. Bot., Res. Inst. Texas 2(1): 70. 2008. Capparis tenuisiliqua Jacq., Enum. Syst. Pl. 24. 1760.

Trees or shrubs, $5 \mathrm{~m}$ tall; stem glabrous or tomentosus on new branch. Leaves simple, alternate, arranged spirally, subtended by a pair of triangular subulate stipules; petioles terete, unequal size, $1-6 \mathrm{~cm}$ long, tomentosus or puberulous, pulvinus present in booth tips. Blades membranaceous to papyraceous, elliptic to elliptic-oblanceolate 8-17 $\times 3-12 \mathrm{~cm}$, base rounded, obtuse and truncate to slightly cordate, acute at apex, margin entire, puberulous beneath and glabrous above on new leaves, glabrous throughout on mature leaves; 8-12 pairs of secondary veins. Racemes terminal, with 20-50 flowers; rachis (sub)terete, ventrally canaliculated and glabrous. Flowers actinomorphic and hermaphroditic, however some of the terminal flowers can be a monoic male flower; subtended by three small triangular bracts; pedicels 4-14 $\mathrm{mm}$ long, terete, glabrous or puberulous to tomentosus. Calyx with open aestivation, sepals fused at the base, forming a small tube, elliptic to deltoid $15-20 \times 1-15 \mathrm{~cm}$, margin ciliate, apex acute, with triangular lobes $1-15 \times 1-2 \mathrm{~mm}$, 
glabrous or puberulent. Floral nectaries scales deltoid and glabrous. Petals imbricated, obovate to oblanceolate, green, 4-10 $\times 4-6 \mathrm{~mm}$, obtuse at apex. Stamens 15-20, filaments exsert, 15-20 mm long, glabrous; anthers basifixed 2-3 mm long. Gynophore exsert, 15-35 mm long, glabrous; ovary cylindric $2-6 \times 0.5-1 \mathrm{~mm}$, glabrous; stigma sessile and truncate. Fruits capsular, torulosus, moniliform, cylindric to oblong 3-20 × 4-5 cm, exocarp smooth; seeds 3-30, elliptic.

Selected specimens examined: COLOMBIA. ANTIOQUIA: Amalfi, sector Almenara, vereda Peldar, 3.X.1992, R. Fonnegra 4537 (COL, HUA). BOLÍVAR: Isla de Tierra Bomba, $10^{\circ} 21^{\prime} 47.18^{\prime \prime} \mathrm{N}, 75^{\circ} 33^{\prime} 47.74^{\prime \prime} \mathrm{W}$, XIII.1996, H. Mendoza 1747 (FMB, JAUM). NORTE DE SANTANDER: San José de Cúcuta, cerro Tasajero, vía San Faustino, $8^{\circ} 2$ '2.16”N, 72²8’11.94”W, 9.VIII.2006, M. Carillo 346 (COL).
Distributed in Colombia and Venezuela. Found mainly in tropical dry forest. However, this species has been registered in wet and Andean forest (Fig. 2d).

\section{Morisonia L.}

Small trees or shrubs, stem stellate, stellateechinate or lepidote-peltate. Leaves simple, alternate, arranged spirally, petioles of unequal length with pulvinus present at both tips. Blades coriaceous, (sub)peltate at base. Racemes cauliflowers or branch flower arrangements, densely stellate or lepidote-peltate. Flowers actinomorphic and hermaphrodite, densely stellate. Stamens insert, biseriate and anther basifixed. Gynophore insert, ovary ovoid and ribbed. Fruits amphisarca, exocarp slightly verrucose.

\section{Identification key to the species of Morisonia}

1. Flowers with more than 40 stamens and ovary ribbed $22-23 \mathrm{~mm}$ long ....... 7.1. Morisonia americana

1'. Flowers with less up to 30 stamens and ovary no ribbed $3-9 \mathrm{~mm}$ long .........7.2. Morisonia multiflora

7.1. Morisonia americana L., Sp. P1. 1: 503. 1753. Morisonia elliptica Rusby., Descr. S. Amer. Pl., Morisonia johnstonii Urb., Symb. Antill. 5: 348. 1907.

Trees or shrubs with scandent branches to $5 \mathrm{~m}$ tall; stem stellate or lepidote-peltate. Petioles terete, $1-14 \mathrm{~cm}$ long, stellate or lepidotepeltate. Blades coriaceous, oblong-elliptic to oblanceolate $8-30 \times 3-15 \mathrm{~cm}$, rounded at base, cordate sub(peltate), obtuse to retuse at apex, margin entire to slightly undulate, glabrous or stellate at the base of the blades in new leaves; 7-10 pairs of secondary veins yellow, ascending, prominent nerves with trichomes translucent stellate-tomentosus in juvenile leaves, glabrous on mature leaves. Corymbous racemes axillar, with 3-20 flowers; rachis angular, ventrally canaliculated and stellate. Flowers actinomorphic and hermaphroditic subtended by a pair of linear bracts, stellate throughout; pedicels angular or terete 1-20 mm long, glabrous, lepidote-peltate. Sepals with closed aestivation, with 2-4 irregular segment triangular 6-8 $\times 6 \mathrm{~mm}$, reflexed, obtuse at apex, echinate outside and glabrous inside. Floral nectaries scales deltoid and stellate. Petals imbricated, obovate to oblanceolate, greenish, 7-10 × 4-6 mm, obtuse at apex, stellate-peltate outside and stellate inside. Stamens 17-30, filament insert, $8-10 \mathrm{~mm}$ long, glabrous; anthers basifixed, 2-3 mm long. Gynophore 1-16 mm long, stellate at base; ovary 3-9 × 2-7 mm, stellate-peltate to tomentosus; stigma sessile and capitated. Fruits oblong, 4-8 cm diameter, exocarp slightly verrucose; seeds $10-30$, reniform to ellipsoidal (Fig. 2d).

Selected specimens examined: COLOMBIA. ATLÁNTICO: Barranquilla, predios de Argos, entre planta Termoflores y Cartón de Colombia, $11^{\circ} 1^{\prime} 41.57^{\prime}$ 'N, $74^{\circ} 48^{\prime} 50.37^{\prime \prime} \mathrm{W}, 11 . I I I .2013$, J.M. VélezPuerta 4450 (MEDEL). SANTANDER: Girón, vereda Chocoa, sector el Puente 74'20.50"N, 73¹1'13.90”'W, 22.VI.2006, C. Díaz 359 (COL).

Distributed from Mexico, Mesoamerica to Colombia, Venezuela, Ecuador to Antilles. It is found at tropical dry forest, wet and Andean forest (Fig. 2d).

7.2. Morisonia multiflora Triana \& Planch. Ann. Sci. Nat., Bot., sér. 4 17: 87. 1862.

Trees or shrubs, 8-15 m tall; stem stellate or lepidote-peltate. Leaves simple, alternate, arranged spirally, petioles terete, $1-10 \mathrm{~cm}$ long, stellate or lepidote-peltate, ventrally canaliculated. Blades coriaceous, elliptic to ovate $8-40 \times 3-25$ $\mathrm{cm}$, cordate or (sub)peltate at base, obtuse at apex, margin entire, glabrous or stellate at blades base in new leaf; 8-9 pairs of secondary veins, 
principal vein stellate-tomentosus in juvenile leaves, scattered or glabrous on mature leaves. Corymbose racemes axillar, with 20-35 flowers, rachis terete, ventrally canaliculated and stellate. Pedicels terete, $6 \mathrm{~mm}$ long, stellate. Sepals with closed aestivation, with 2-4 irregular segment, deltoid, $10 \times 4-8 \mathrm{~mm}$, rounded at apex, stellate tomentosus on both sides. Floral nectaries scales deltoid and densely stellate throughout. Petals imbricated, obovate to oblong, greenish, 17-19 $\times 10-15 \mathrm{~mm}$, rounded at apex, stellate-peltate on both sides. Stamens $45-50$, filaments insert, 14-15 mm long, stellate tomentosus; anthers basifixed, 2-3 mm long. Gynophore 1-16 mm long, peltate- stellate throughout; ovary 12-13 $\times$ 5-7 mm, stellate-peltate to tomentosus; stigma sessile and capitated. Fruits globoids, $5-8 \mathrm{~cm}$ diameter, exocarp slightly verrucose; seeds 10-30 ovoid.

Selected specimens examined: COLOMBIA. ANTIOQUIA: Amalfi, sector Almenara, 1,007 m, 25.VI.2003, A. Yepes 48 (HUA). Puerto Boyacá, Puerto Romero, 10.III.2000, C. Bernal 385 (COL). SANTANDER: Cimitarra, Puerto Olaya, $6^{\circ} 28^{\prime} 2.85^{\prime}$ N , 74²1'1.16”'W, 1.XII.1999, A. Idarraga 1286 (COL).
Distributed in Colombia. Found mainly in tropical dry forest and occasionally in wet forest (Fig. 2d).

\section{Preslianthus Iltis \& Cornejo.}

Trees or shrubs stellate. Leaves simple, alternate and arranged in spiral, petiole of similar length, ventrally canaliculated, and pulvinus inconspicuous. Blades coriaceous to chartaceous, margin revolute or slightly revolute, stellate beneath and glabrous above, apex acuminate to acute. Inflorescences terminal, panicles with 6 flowers for each panicle; rachis terete, ventrally canaliculated, densely stellate, reddish or transparent. Flowers actinomorphic and hermaphroditic, pedicels angular, ventrally canaliculated, and densely stellate reddish or transparent. Floral nectaries, episepals glands, scales deltoid and densely stellate throughout. Sepals valvate, stellate throughout. Petals imbricates of similar length of sepals, white or white-greenside. Stamens numerous very exsert, glabrous, anther dorsifixed. Gynophore very exsert; ovary glabrous. Fruits in amphisarca, globoid to oblongoid.

\section{Identification key to the species of Preslianthus}

1. Sepals and petals larger than a $6 \mathrm{~mm}$ long and fruits elongates

8.1. Preslianthus detonsus

1'. Sepals and petals smaller than a $5 \mathrm{~mm}$ long and fruits globoids 8.2. Preslianthus pittieri

8.1. Preslianthus detonsus (Triana \& Planch.) Iltis \& Cornejo, Harvard Pap. Bot. 16(1): 70. 2011. Capparis detonsa Triana \& Planch., Ann. Sci. Nat., Bot., série 4 17: 80. 1862.

Trees or shrubs, 3-15 m tall; stem glabrous, on new branch densely stellate, brown or reddish. Petioles 3-5 cm long, (sub) terete or slightly angular and stellate reddish. Blades coriaceous to chartaceous, oblong, elliptic to ovate, $24-26 \times 10 \mathrm{~cm}$, rounded to cuneate at base; acuminate at apex, margin revolute, reddish stellate on both sides in juvenile leaves, while on mature leaves are scattered or glabrous; 10 pairs of secondary veins. Rachis angular, densely stellate; pedicels 2.9-4 mm long, angular and densely stellate. Sepals deltoid $6 \times 3-4 \mathrm{~mm}$, apex acute to obtuse, reddish, stellate tomentosus outside and glabrous inside. Petals yellow, oblanceolate to deltoid 9-10 $\times 7 \mathrm{~mm}$, apex rounded and glabrous. Stamens 30-36, filament exsert 15-16 mm long, glabrous; anthers basifixed of $4 \mathrm{~mm}$ long. Gynophore exsert 30-70 mm long, glabrous; ovary oblongoid 5-10 × 5-7 mm, glabrous; stigma sessile and truncated. Fruits in amphisarca, oblongoid 6-14 × 5-8 cm, exocarp smooth; seeds 6-20 elliptic to ovoid.

Selected specimens examined: COLOMBIA. AMAZONAS: Leticia, camino hacia Tarapacá, km 17, 17.XII.1965, G. Lozano 432 (COL). CAQUETÁ: Cartagena, 20.VI.1953, Romero-Castañeda $R 4040$ (COL) CHOCÓ: al norte de Bahía Solano, 6¹6’00”N 77019'00'W, 10.I.1984, A. Juncosa 1874 (JAUM). CÓRDOBA: Tierralta, Isla Boca de las Mujeres, 0806'36”'N, 76009'31'”, 28.XI.1999, N. López 4642 (JAUM).

Distributed in Colombia, Venezuela and Brazil. It is found in cloud forests, pre-mountain jungle, rainforest and disturbed forest (Fig. 3a). 
8.2. Preslianthus pittieri (Standl.) Iltis \& Cornejo., Harvard Pap. Bot. 16(1): 70. 2011. Capparis pittieri Standl., J. Wash. Acad. Sci. 17(10): 253. 1927. Capparis crotonantha Standl., Publ. Field Mus. Nat. Hist., Bot. Ser. 4(8): 210. 1929. Capparis schunkei J.F. Macbr., Publ. Field Mus. Nat. Hist., Bot. Ser. 4(7): 170. 1929.

Trees or shrubs, 3-12 $\mathrm{m}$ tall; stem glabrous, on new branch densely stellate, brown or reddish. Petioles (sub)terete or slightly angular, of similar length $10-11 \mathrm{~cm}$ long, stellate reddish. Blades coriaceous to chartaceous, oblong, elliptic to ovate $15-29 \times 5-10 \mathrm{~cm}$, base rounded to cuneate; acuminate at the apex, margin entire or slightly revolute, reddish stellate on both sides in juvenile leaves while on mature leaves are scattered or glabrous; $10-12$ pairs of secondary veins. Rachis terete, densely stellate, reddish or transparent. Pedicels 7-9 $\mathrm{mm}$ long, terete, densely stellate, reddish or transparent. Sepals valvate, deltoid $2-3 \times 1$ $\mathrm{mm}$, apex acute, transparent or slightly reddish stellate tomentosus outside and glabrous or stellate inside. Petals white, oblanceolate to deltoid 3-3.5 $\times 3 \mathrm{~mm}$, apex acute, transparent stellate tomentosus outside and glabrous inside. Stamens 16-25, filament exsert 15-40 $\mathrm{mm}$ long, glabrous; anthers basifixed of 1-2 $\mathrm{mm}$ long. Gynophore exsert 15-50 mm long, glabrous; ovary oblongoid $1-3 \times 1-1.5 \mathrm{~mm}$, glabrous, stigma sessile and truncated. Fruits in amphisarca, ellipsoidal 5-8 diameter, exocarp smooth; seeds 10-30, reniform to ovoid.

Selected specimens examined: COLOMBIA. CALDAS: Norcasia, Quiebra de Roque, 20.III.2012, J. Ramírez 247 (JAUM). CHOCÓ: El Carmen, alrededores de Bahía Solano, 6¹3'09"N, 77²4'28”'W, 7.V.1992, C. Barbosa 6990 (FMB, HUA). CÓRDOBA: Tierralta, quebrada Naim, 0806'36'N, 7609'31'”W, 29.XI.1999, N. López 4676 (JAUM). SANTANDER: Puerto Parra, 6 50 '24.8' N, 7400'28.4"'W, 29.VIII.2010, A. Cogollo 12749 (JAUM).

Distributed in Colombia, Venezuela, Brazil and Bolivia. It is found in cloud forests, pre-mountain jungle, rainforest and disturbed forest (Fig. 3a).

\section{Quadrella (DC.) J. Presl.}

Small trees or shrubs; densely lepidotepeltate to stellate. Leaves simple, alternate, arranged spirally; petioles of similar length, ventrally canaliculated. Inflorescences terminal or axillar, racemes umbelated, or panicles. Flowers actinomorphic and hermaphroditic, subtended by a pair of subulate bracts, pedicels angular ventrally canaliculated. Floral nectaries, episepals glands scuamiform. Petals imbricated. Gynophore developed or not. Fruits capsular torulous, linear-cylindric.

\section{Identification key to the species of Quadrella}

1. Blades silver or bronze, acuminate to acute at the apex and gynophore present.

9.1. Quadrella indica

1'. Blades brown to reddish, emarginated, rounded to apiculate at the apex and gynophore absent. 9.2. Quadrella odoratissima

9.1. Quadrella indica (L.) Iltis \& Cornejo., J. Bot. Res. Inst. Texas 4(1): 126-127. 2010. Breynia indica L., Sp. P1. 1: 503. 1753. (1.V.1753). Capparis amygdalina Lam., Encycl. 1: 608. 1783[1785]. Capparis furfuracea Ruiz \& Pav. ex DC., Prodr. 1: 252. 1824. Capparis furfuracea Sessé \& Moc., Fl. Mex. 129. 1894. Capparis indica (L.) Druce., Bot. Exch. Club Soc. Brit. Isles 3: 415. 1914. Capparis tonduzii Briq Annuaire Conserv. Jard. Bot. Genève 17: 391-392. 1914. Linnaeobreynia indica (L.) Hutch., Gen Fl. Pl. 2: 311. 1967. Linnaeobreynia tonduzii (Briq.) Hutch., Gen Fl. Pl. 2: 310. 1967. Pleuteron breynia
(L.) Raf., Syl. Tellur. (4): 109. 1838. Pseudocroton tinctorius Müll., Arg. Flora 55(1): 24-25. 1872. Quadrella breynia (L.) J. Presl., Přir. Rostlin 2: 261. 1825. Quadrella furfuracea (Ruiz \& Pav. ex DC.) J. Presl., Přir. Rostlin 2: 260. 1825. Uterveria breynia (L.) Horti Bonon. Pl. Nov 2: 10. 1839.

Trees or shrubs, 3-10 m tall; stem glabrous, lepidote-peltate, densely on new branch, silver or slightly bronze. Petioles $2-6 \mathrm{~cm}$ long, angular, silver lepidote-peltate. Blades membranous, elliptic, oblong, oblongate-elliptic to obovate $4-11 \times 3-6 \mathrm{~cm}$, base cuneate and obtuse, acute to mucronated at the apex, margin entire or 

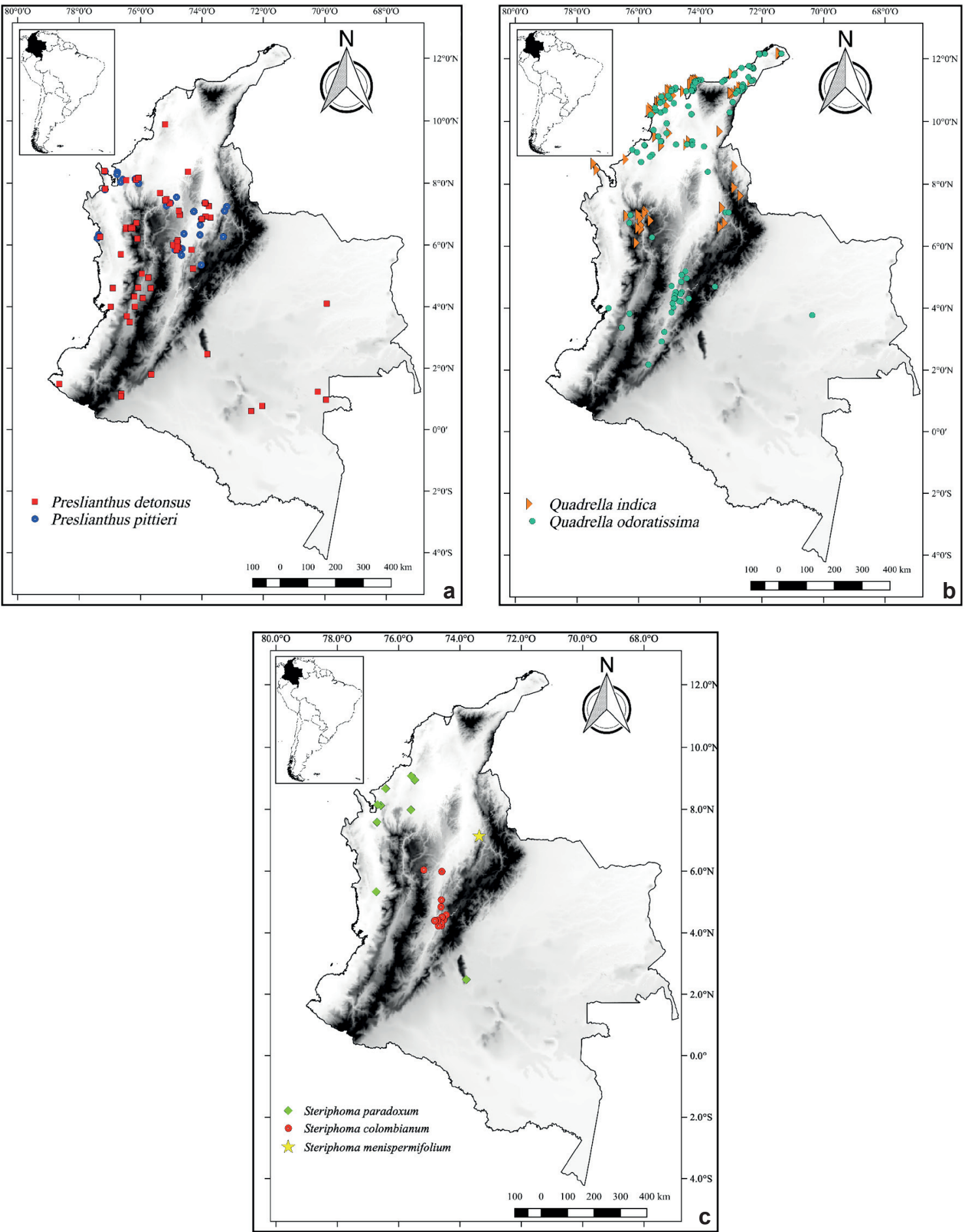

Figure 3 - Distribution map of species of Capparaceae to the flora of Colombia - a. P. detonsus and P. pittieri; b. Q. indica and Q. odoratissima; c. S. colombianum, S. menispermifolium and S. paradoxum. 
slightly undulate, silver to slightly bronze beneath and stellate above; with 18-20 pairs lateral nerves. Inflorescences axillar, corymbose or umbellate with 12-20 flowers; rachis terete, densely lepidote-peltate. Pedicels 5-10 mm long, and densely lepidote-peltate. Sepals fused in the base, deltoid 2-3 $\times 1-2 \mathrm{~mm}$, apex acute, peltate outside and stellate tomentosus inside. Floral nectaries, erect deltoid scales and densely stellate tomentosus throughout. Petals cream, orbicular to elliptic 7-13 × 4-8 mm, apex acute, silver lepidote-stellate in booth sides. Stamens 15-30, filament cream 7-13 mm long, exsert, stellate at the base; anthers dorsifixed of 2 $\mathrm{mm}$ long. Gynophore 30-35 mm long, insert, lepidote-peltate throughout; ovary cylindric 3-5 $\times 1-2 \mathrm{~mm}$, densely lepidote-peltate throughout; stigma sessile and capitated. Fruits capsular, bacciform, torulous, cylindric 4-24 × 1-2 cm, exocarp smooth; seeds 2-11, globoid.

Selected specimens examined: COLOMBIA. ANTIOQUIA. Briceño, cuenca de Tenche, Río Cauca, $7^{\circ} 7^{\prime} 36.59^{\prime \prime} \mathrm{N}, 75^{\circ} 39^{\prime} 48.88^{\prime \prime} \mathrm{W}, 13 . X I I .2012, D$. Zapata 24 (COL, JAUM). LA GUAJIRA: Distracción, sitio Las Casitas-El Socorro, $10^{\circ} 53^{\prime} 51.80^{\prime} \mathrm{N}$, $72^{\circ} 51$ '58.08”W, 31.VIII.1990, Marulanda O et al. 2110 (HUA). SUCRE: Colosó, Primates, 9³1'40.49”'N, 75² $1^{\prime} 54.18^{\prime \prime} \mathrm{W}, 16 . \mathrm{III} .1997$, L. Prado 091 (FMB).

Distributed from Mesoamerica to north of Colombia. Found in tropical dry forest (Fig. $3 b)$.

9.2. Quadrella odoratissima (Jacq.) Hutch., Gen. Fl. Pl. 2: 308. 1967. Capparis odoratissima Jacq., P1. Hort. Schoenbr. 1: 57-58, t. 110. 1797. Quadrella intermedia (Kunth) J. Presl., Prrir. Rostlin 2: 261. 1825. Capparis intermedia Kunth., Nov. Gen. Sp. (quarto ed.) 5: 98. 1821. Capparis ferruginea L., Syst. Nat. (ed. 10) 2: 1071. 1759. Colicodendron lepidotum Turcz., Bull. Soc. Imp. Naturalistes Moscou 27(2): 327. 1854. Capparis lepidota (Turcz.) Knuth, Repert. Spec. Nov. Regni Veg. Beih.43: 347. 1927

Trees or shrubs, 3-30 m tall; stem glabrous, lepidote-peltate, densely on new branch, bronze. Petioles angular to sub(terete), 4-15 cm long, bronze lepidoted-peltated. Blades coriaceous, elliptic, oblong to obovate $4-11 \times 1-6 \mathrm{~cm}$, base rounded, cuneate, obtuse, rounded, apex emarginated to apiculate, bronze lepidotepeltate beneath and glabrous above; with $6-8$ pairs of secondary veins. Inflorescences axillar or terminal, corymbose racemes with 8-12 flowers; rachis angular, ventrally canaliculated and densely lepidote-peltate. Pedicels 5-10 $\mathrm{mm}$ long, smooth or densely lepidote-peltate. Sepals valvate, oblong to ellipsoidal 4-6 $\times$ 2-4 mm, apex acute to obtuse, stellate outside and lepidote-peltate inside. Floral nectaries, scales deltoid and glabrous. Petals white to purple, oblong to elliptic 5-7 $\times 4-6 \mathrm{~mm}$, apex acute, middle line of lepidote-peltate trichomes outside and glabrous inside. Stamens 15-40, filament slightly exsert, 4-9 $\mathrm{mm}$ long, short pilose at the base; anthers dorsifixed of $2 \mathrm{~mm}$ long. Gynophore not developed, pistil short, insert, densely lepidote-peltate throughout; ovary cylindric 3-6 3 2-4 mm, densely peltate throughout; stigma sessile and capitated. Fruits capsular, bacciform, torulous, oblong to cylindric 2-2 2 1-2 cm, exocarp lepidote; seeds $2-11$, ovoid and arranged in two rows.

Selected specimens examined: COLOMBIA. ARCHIPIÉLAGO DE SAN ANDRÉS, PROVIDENCIA Y SANTA CATALINA: Isla de Providencia, III.1977, J. Freeman 12 (COL, MEDEL, UTMC). CESAR: Chimichagua, $9^{\circ} 15,15.8 ” \mathrm{~N}$, 7548'47”W, 03.III.2007, N. Jiménez 018 (COL). SANTANDER: Girón, Tigreros-Cruces, $7^{\circ} 5^{\prime} 21.84^{\prime}$ N, $3^{\circ} 10^{\prime} 44.03$ ”'W, 31.V.2015, H. David 4923 (CDMB). VALLE DEL CAUCA: Buenaventura, Bajo Calima, Estación de Silvicultura San Isidro, XI.1987, C. Barbosa 73 (FMB).

Distributed in Central America, the Antilles, Colombia and Venezuela. Found in tropical dry forest, and less common in wet forest (Fig. 3b).

\section{Steriphoma Spreng.}

Small trees or shrubs with stem stellate. Leaves simple, alternate, arranged spirally, petioles angular to terete, unequal length, densely stellate throughout, pulvinus at the both sides. Blades membranaceus to papyraceous, stellate beneath and glabrous above, base acute, such as apex, and margin entire. Flowers zygomorphic and hermaphroditic, erect, subtended by a linear stellate, deciduous bracts, when fall, remains at the base in the form of a triangular bract. Pedicels angular, ventrally canaliculated. Petals valvate, Floral nectaries, episepals glands. Stamens exsert such as gynophore. Fruits capsular, and torulous. 


\section{Identification key to the species of Steriphoma}

1. Small trees, blades of base obtuse or acute, rachis no ventrally canaliculated ..................................2

2. Blades of margin revolute, inflorescences with less of 39 flowers, and flowers with pedicel 10-15 mm long..... 10.1. Steriphoma colombianum

2'. Blades of margin entire, inflorescences with more than 41 flowers, and flowers with pedicel 30-50 mm long 10.3. Steriphoma paradoxum

1'. Climbing, blades of base truncate to cordate ventrally canaliculated.

10.2. Steriphoma menispermifolium

10.1. Steriphoma colombianum Dugand. Caldasia 3(12): 149. 1944.

Small trees or shrub, $3 \mathrm{~m}$ tall; stem glabrous or stellate on new branch. Petioles 2-6 cm long. Blades papyraceous or coriaceous, ovatelanceolated $10-15 \times 3.3-5 \mathrm{~cm}$, base attenuate to rounded, apex acuminate, margin revolute, stellate-tomentosus beneath and glabrous above; with $8-10$ pairs of lateral nerves. Inflorescences terminal, racemes with 40 flowers; rachis terete, densely stellate tomentosus, bronze. Pedicels 10-25 mm long, densely covered for bright orange trichomes. Sepals $13 \mathrm{~mm}$ long, fused, forming a hypanthium, 3-4 lobes triangular to deltoid $6 \times 2$ $\mathrm{mm}$, apex acute to obtuse, outside densely covered by bright orange stellate trichomes, glabrous inside. Floral nectaries, scales deltoid and glabrous. Petals valvate, yellow, oblong to oblanceolate $18 \times 4 \mathrm{~mm}$, apex acute, stellate outside and glabrous inside. Stamens 6, filament very exsert 70-80 $\mathrm{mm}$ long, curved, glabrous; anthers basifixed of $7-8 \mathrm{~mm}$ long. Gynophore very exsert of $80-90 \mathrm{~mm}$ long, densely stellate at the base; ovary oblong-cylindric 12-20 $\times 2 \mathrm{~mm}$, stellate and pink; stigma sessile, discoid and capitated. Fruits berry, torulous 7-20 × 2-4 $\mathrm{cm}$, exocarp smooth; seeds 13-16, oblong - elliptic. Selected specimens examined: COLOMBIA CUNDINAMARCA: Quebrada Camargo, North of Apulo, $480 \mathrm{~m}, 4^{\circ} 30^{\prime} 40.70^{\prime \prime} \mathrm{N}, 4^{\circ} 35^{\prime} 12.23^{\prime \prime} \mathrm{W}$, 05.V.1944, E.P. Killip 38238 (COL). TOLIMA: Melgar, vereda La Primavera, Reserva Natural La Victoria, 4¹4'21.6”'N, 74³5'55.3”W, 20.VI.2015, N. Marín 1931 (COL).

Endemic of Colombia. It is found in lowland wet forest and Andean forest (Fig. 3c).

10.2. Steriphoma menispermifolium Cornejo \& Mercado-Gómez., Revista Cient. Cien. Nat. Ambien. 10(1): 1, f. 1. 2016.

Climbing to $3 \mathrm{~m}$ long; stem glabrous or stellate on new branch. Petioles 3-6 cm long. Blades membranaceous to papyraceous, ovatelanceolate $5-12 \times 3-5 \mathrm{~cm}$, base truncate to strongly cordate, apex acute to acuminate, margin entire, stellate tomentosus beneath and short pilose above; with 5-7 pairs of secondary veins. Inflorescences terminal, racemes with 40 flowers; rachis (sub) terete to slightly canaliculated, densely stellate tomentosus, brown. Pedicels 25-50 mm long, sub-erect, densely covered for trichomes bright orange. Sepals $20-23 \mathrm{~mm}$ long fused forming a hypanthium, 3-4 lobes triangular to deltoid 8-9 $\times 1-4 \mathrm{~mm}$, apex acute to obtuse, densely covered by stellate trichomes bright orange outside and glabrous inside. Floral nectaries, scales deltoid and glabrous. Petals yellow, oblong $24 \times 6 \mathrm{~mm}$, apex acute, stellate outside and glabrous inside. Stamens 6, filament 55-70 mm long, very exsert, curved, glabrous; anthers basifixed of 2-4 mm long. Gynophore 60-95 mm long, very exsert, densely stellate at the base; ovary oblong-cylindric $12-20 \times 2 \mathrm{~mm}$, stellate and pink; stigma sessile, discoid and capitate.

Selected specimens examined: COLOMBIA. SANTANDER: Girón, vereda Marta, $7^{\circ} 08^{\prime} 10.9^{\prime}$ N, 73²2'12.9”W, VI.2013, J. Castro 275 (HUA).

Endemic of Colombia. It is found in tropical dry forest of inter Andean valley of river Sogamoso (Santander) (Fig. 3c).

10.3. Steriphoma paradoxum (Jacq.) Endl., Flora 15(2): 396. 1832. Capparis paradoxa Jacq., Pl. Hort. Schoenbr. 1: 58, t. 111. 1797. Roemera paradoxa (Jacq.) Tratt., Gen. P1. 88. 1802. Stephania cleomoides Willd., Sp. Pl. 2(1): 239. 1799. Steriphoma cleomoides Spreng., Syst. Veg. [Sprengel] 4(2): 139. 1827. Steriphoma paradoxum fo. venezuelanum (Briq.) Steyerm., Fieldiana, Bot. 28(1): 239. 1951. Steriphoma paradoxum subsp. venezuelanum (Briq.) Cornejo., Anales Jard. Bot. Madrid 63(2): 251. 2006. Steriphoma venezuelanum Briq., Annuaire Conserv. Jard. Bot. Genève 17: 394-395. 1911-1913.

Small trees or shrubs to $3 \mathrm{~m}$ tall; stem glabrous or stellate on new branch. Petioles $3.5-11 \mathrm{~cm}$ long. Blades membranaceous to 
papyraceous, ovate-lanceolated $12-30 \times 4-10$ $\mathrm{cm}$, base attenuate, rounded to asymmetrical, apex acuminate, margin entire, stellate-tomentosus beneath and glabrous above; 8-10 pairs of secondary veins. Inflorescences terminal, racemes with 40-60 flowers, rachis terete, densely stellate tomentosus, bronze. Pedicels 30-50 mm long densely covered by trichomes bright orange. Sepals fused $14 \mathrm{~mm}$ long, forming a hypanthium, 3-4 lobes triangular to deltoid 9-10 × 2-6 mm, apex acute, densely covered by stellate trichomes bright orange outside and glabrous inside. Floral nectaries, scales deltoid and glabrous. Petals yellow, oblong to oblanceolate $23 \times 3 \mathrm{~mm}$, apex acute, stellate outside and glabrous inside. Stamens 6, filament very exsert 30-80 mm long, curved, glabrous; anthers basifixed of 7-8 mm long. Gynophore very exsert 80-90 mm long, glabrous; ovary oblong-cylindric $12-20 \times 2 \mathrm{~mm}$, densely stellate and pink; stigma sessile, discoid and capitate. Fruits berry, torulous 7-20 × 2-4 cm, exocarp smooth, seeds 13-16, oblong - elliptic.

Selected specimens examined: COLOMBIA. ANTIOQUIA: Chigorodo, Barranquillita, 18.III.1948, Ruiz E ns. (MEDEL). CÓRDOBA: Chima, vereda El Cerro, 9॰4'58'N, 75³5'13'W, 08.VI.2005, O. RiveraDíaz 2539 (COL).

Distributed from Mexico to Perú. It is found in lowland wet forest and Andean forest (Fig. 3c).

\section{Acknowledgment}

We wish to thanks the directors and curators of the herbaria visited. We thank Xavier Cornejo for his commentaries in nomenclature and taxonomic history and J. Richard Abbott for his useful comments to the manuscript. We thank Jorge Velez by his support on literature and taxonomic discussions. The first author thanks COLCIENCIAS and "Universidad de SUCRE" for the financial support of his doctoral studies.

\section{References}

Aguirre De la Hoz AC \& Zapata TR (2017) Capparaceae Juss. del departamento del Atlántico, Colombia. Ciencia en Desarrollo 8: 51-69.

Bentham G \& Hooker J (1862) Genera Plantarun. Herbariis kewensibus servata, Londres. 454p.

Bernal RS, Gradstein R \& Celis M (2016) Capítulos introductorios - Líquenes a Lytrhaceae. In: Bernal R, Gradstein SR \& Celis M. Catálogo de plantas y líquenes de Colombia. Bogotá. Instituto de Ciencias Naturales, Universidad Nacional de Colombia: 1-1500.
Bullock A (1958) Indicis Nominum Familiarum Angiospermarum Prodomus. Taxon 7: 35.

Bullock A (1959) Nomina Familiarum Conservanda Proposita. Taxon 8: 154-181.

Cornejo X (2010) Colicodendron martianum (Capparaceae), a remarkable new species from northeastern Brazil. Harvard Papers in Botany 15: 103-106.

Cornejo X \& Iltis H (2005a) Studies in the Capparaceae XXIII: Capparis coimbrana, a new species from Bolivia. Brittonia 57: 155-161.

Cornejo X \& Iltis H (2005b) Studies in the Capparaceae XXVI. Capparis bonifaziana, a new species and western Ecuadorian sister to the mostly Amazonian C. macrophylla. Novon 15: 393-404.

Cornejo X \& Iltis H (2006) New combinations in Capparaceae sensu stricto for flora of Ecuador. Harvard Papers in Botany 11: 17-18.

Cornejo X \& Iltis H (2008a) New combinations in South American Capparaceae. Harvard Papers in Botany 13: 117-120.

Cornejo X \& Iltis H (2008b) The reinstatement of Capparidastrum (Capparaceae). Harvard Papers in Botany 13: 229-236.

Cornejo X \& Iltis H (2008c) A revision of Colicodendron (Capparaceae). Journal of the Botanical Research Institute of Texas 2: 75-93.

Cornejo X \& Iltis H (2008d) A revision of the American species of the genus Crateva (Capparaceae). Harvard Papers in Botany 13: 121-135.

Cornejo X \& Iltis H (2008e) Two new genera of Capparaceae: Sarcotoxicum and Mesocapparis stat. nov., and the reinstatement of Neocalyptrocalyx. Harvard Papers in Botany 13: 103-116.

Cornejo X \& Iltis H (2009) Hispaniolanthus: a new genus of Capparaceae endemic to Hispaniola. Harvard Papers in Botany 14: 9-14.

Cornejo X \& Iltis H (2010a) Capparidastrum tuxtlense (Capparaceae), a new Mexican endemic. Brittonia 62: $120-125$.

Cornejo X \& Iltis H (2010b) Studies in Capparaceae XXVII: six new taxa and new combination in Quadrella. Journal of the Botanical Research Institute of Texas 4: 75-91.

Cornejo X \& Iltis H (2010c) Lectotypification and a new combination in Cynophalla (Capparaceae). Rodriguésia 61: 153-155.

Cornejo X \& Iltis H (2010d) Three new species of Capparidastrum (Capparaceae) from Colombia, Venezuela, and Ecuador. Harvard Papers in Botany 15: $155-163$.

Cornejo X \& Iltis H (2012) Capparaceae In: Carvajal S \& Gonzales-Villareal LM (eds.) La flora de Jalisco y áreas colindantes. Jalisco Saulo A. Cortez Arévalo-Orgánica Editores. Pp. 1-71.

Cornejo X \& Iltis H (2013) New combinations in Capparaceae for Flora Mesoamericana. Harvard Papers in Botany 18: 147. 
Cornejo X, Iltis H \& Cerón CE (2014) Capparidastrum tafallanum (Capparaceae), a new species from the northwestern Andean slopes of Ecuador. Harvard Papers in Botany 19: 189-191.

Cornejo X, Iltis H \& Tomb AS (2008) Anisocapparis y Monilicarpa: dos nuevos géneros de Capparaceae de América del Sur. Journal of the Botanical Research Institute of Texas 2: 61-74.

Cornejo X \& Mercado-Gómez J (2016) Steriphoma menispermifolia: una nueva especie de Capparaceae de Colombia. Revista Científica Ciencias Naturales y Ambientales 10: 1-3.

Cronquist A (1982) An integrated system of classification of flowering plants. Columbia University Press, New York. 1262p.

Crosswhite S \& Iltis H (1966) Studies in the Capparidaceae X: orthography and conservation: Capparidaceae vs. Capparaceae. Taxon 15: 205-214.

De Candolle AP (1824) Ordo XII. Capparidae. Juss. In: de Candolle AP (ed.) Prodromus systematis naturalis regni vegetabilis, sive, Enumeratio contracta ordinum generum specierumque plantarum huc usque cognitarium, juxta methodi naturalis, normas digesta auctore. Sumptibus Sociorum Treuttel et Wurtz, Parisii. Pp. 237-254.

Dugand A (1941) El género Capparis en Colombia. Caldasia 2: 29-54.

Dugand A (1944) Capparidaceae: Stubelia Pax, sinónimo de Balencita Karsten. Caldasia 2: 371-373.

Dugand A (1968a) Acerca de unas Capparis de la flora colombiana. Caldasia 10: 219-229.

Dugand A (1968b) Por la conservación de "Capparidaceae" y no "Capparaceae". Caldasia 47: $215-217$.

Eichler A (1865) Capparideae. In: Martius CFP \& Eichler AG (eds.) Flora brasiliensis. Lipsiae. Pp. 238-292.

Galetti LA, Mogni VY, Oakley LJ, Pennington T \& Prado DE (2016) Cynophalla polyantha (Capparaceae), nuevo registro para la flora de Argentina. Boletín Sociedad Argentina de Botánica 2: 373-378.

Hall JC (2008) Systematics of Capparaceae and Cleomaceae: an evaluation of the generic delimitations of Capparis and Cleome using plastid DNA sequence data. Botany 86: 682-696.

Hall JC, Sytsma KJ \& Iltis H (2002) Phylogeny of Capparaceae and Brassicaceae based on chloroplast sequence data. American Journal of Botany 89: 1826-1842.

Holmgren P, Holmgren N \& Barnett L (1990) Index herbariorum. Part I: the herbaria of the world. $8^{\text {th }}$ ed. International Association for Plant Taxonomy, The New York Botanical Garden, New York. 693p.

Hutchinson J (1967) The genera of flowering plants. Oxford University Press, Londres. 659p.

Iltis H (1965) Studies in the Capparidaceae IX. Capparis pachaca and C. oxysepala: taxonomy and geography. The Southwestern Naturalist 10: 57-64.

Iltis H (2005). Studies in the Capparaceae XXIV: Edward Palmer in Corumbá, Brazil and the first collection of Capparis coimbrana. Brittonia 57: 162-166.

Iltis H \& Cornejo X (2007a) Studies in the Capparaceae XXX: Capparicordis, a new genus from the Neotropics. Brittonia 59: 245-254.

Iltis H \& Cornejo X (2007b) Studies in the Capparaceae XXXII: a new combination in Quadrella. Novon: a journal for botanical nomenclature 17: 452-453.

Iltis H \& Cornejo X (2010a) Studies in Capparaceae XXVIII: the Quadrella cynophallophora complex. Journal of the Botanical Research Institute of Texas 4: 93-115.

Iltis H \& Cornejo X (2010b) Studies in the Capparaceae XXIX: synopsis of Quadrella, a Mesoamerican and West Indian genus. Journal of the Botanical Research Institute of Texas 4: 117-132.

Iltis H \& Cornejo X (2016) Capparaceae. In: Bernal R, Gradstein R \& Celis M. Catálogo de plantas y líquenes de Colombia (eds.) Universidad Nacional de Colombia (sede Bogotá). Facultad de Ciencias. Instituto de Ciencias Naturales, Bogotá. Pp. 1034-1037.

Iltis H, Hall JC, Cochrane TS \& Sytsma KJ (2011) Studies in the Cleomaceae I. On the separate recognition of Capparaceae, Cleomaceae, and Brassicaceae. Annals of the Missouri Botanical Garden 98: 28-36.

Judd WS, Sanders RW \& Donoghue MJ (1994) Angiosperm family pairs: preliminary phylogenetic analyses. Harvard Papers in Botany 1: 1-51.

Lanjow J \& Sprague T (1947) International rules of botanical nomenclature. Brittonia 6: 1-120.

Morton CM, Karol KG \& Chase MW (1997) Taxonomic affinities of Physena (Physenaceae) and Asteropeia (Theaceae). Botanical Review 63: 231-239.

Neto RLS, Magalhães FÁL, Tabosa FRS, Moro MF, Silva, MBC \& Loiola MIB (2014) Flora do Ceará, Brasil: Capparaceae. Rodriguésia 65: 671-684.

Neto RLS, \& Jardim JG (2015) Capparaceae no Rio Grande do Norte, Brasil. Rodriguésia 66: 847-857.

Pax F (1891) Capparidaceae. In: Engler A \& Prantl KA (eds.) Die naturlichen Pflanzenfamilien. Verlag von Wilhelm Engelmann, Leipzig. Pp. 209-236.

Pax F \& Hoffmann K (1936) Capparidaceae. In: Engler \& Prantl (eds.) Die Naturlichen Pflanzenfamilien. Engelmann, Leipzig. Pp. 146-233.

Quattrocchi U (2000) CRC world dictionary of plant names. Common names, scientific names, eponums, synonyms, and etymology (A-C). CRC Press LLC, Boca Raton. 523p.

Rodman JE, Karol KG, Price, RA \& Sytsma KJ (1996) Molecules, morphology, and Dahlgren's expanded order Capparales. Systematic Botany 21: 289-307.

Rodman J, Price R, Karol K, Conti E, Sytsma K \& 
Palmer J (1993) Nucleotide sequences of the $r b c L$ gene indicate monophyly of mustard oil plants. Annals of Missouri Botanical Garden 80: 686-699.

Rodríguez-Rodríguez EF, Bussmann, RW, ArroyoAlfaro SJ \& López-Medina SE (2007) Capparis scabrida (Capparaceae) una especie del Perú y Ecuador que necesita planes de conservación urgente. Arnaldoa 14: 269-282.

Short PS (2011) Capparaceae In: Short PS \& Cowie ID (eds.) Flora of the Darwin region. Northern
Territory Herbarium, Palmerston. Pp. 1-24.

Takhtajan A (1997) Diversity and classification of flowering plants. Columbia University Press, New York. 620p.

Thiers B [continuously updated] Index Herbariorum: a global directory of public herbaria and associated staff. New York Botanical Garden's Virtual Herbarium. Available at $<$ http://sweetgum.nybg. org/science/ih/> . Access on Jaunary 2018. 